\title{
THE CENTER OF $\mathrm{SL}_{2}$ TILTING MODULES
}

\author{
DANIEL TUBBENHAUER AND PAUL WEDRICH
}

\begin{abstract}
In this note we compute the centers of the categories of tilting modules for $G=\mathrm{SL}_{2}$ in prime characteristic, of tilting modules for the corresponding quantum group at a complex root of unity, and of projective $G_{g} T$-modules when $g=1,2$.
\end{abstract}

\section{INTRODUCTION}

Let $\mathbb{K}$ denote an algebraically closed field and Tilt $=\operatorname{Tilt}\left(\mathrm{SL}_{2}(\mathbb{K})\right)$ the additive $\mathbb{K}$-linear category of (left-)tilting modules for the algebraic group $\mathrm{SL}_{2}(\mathbb{K})$.

In this note we compute the (categorical) center $\mathbf{Z}$ (Tilt) of Tilt, using the explicit description of the Ringel dual of $\mathrm{SL}_{2}(\mathbb{K})$ from [TW19]. In characteristic zero we have $\mathbf{Z}($ Tilt $) \cong \prod_{\mathbb{N}} \mathbb{K}$ since Tilt is semisimple with simple objects indexed by $\mathbb{N}$. Hence, our main concern is the case of prime characteristic. Thus, for the duration, $\mathbb{K}$ is of characteristic $p \geq 2$.

Theorem A We have isomorphisms of $\mathbb{K}$-algebras

$$
\mathbf{Z}(\text { Tilt }) \cong \mathbb{K}\left[X_{v} \mid v \in \mathbb{N}\right] /\left\langle X_{v} X_{w} \mid v, w \in \mathbb{N}\right\rangle .
$$

We will provide an explicit isomorphism in Theorem 4.6 and the discussion following it. For a possible interpretation of the central elements $X_{v}$ via Donkin's tensor product theorem see Section 4C.

Finally, in Section 5 we compute the centers of the categories of tilting modules in the quantum group case, see Theorem 5.2, and of projective $G_{g} T$-modules for $g=1,2$, see Theorem 5.3, both for $\mathrm{SL}_{2}$.

Acknowledgments. We like to thank Geordie Williamson for bringing the question about the center of tilting modules to our attention, and for helpful exchanges of emails. We also thank Henning Haahr Andersen and Catharina Stroppel for valuable email discussions and comments, and a referee for comments on a draft of this paper.

D.T. was sponsored by a chair in a random hotel during this project. P.W. was supported by the National Science Foundation under Grant No. DMS-1440140, while in residence at the Mathematical Sciences Research Institute in Berkeley, California, during the Spring 2020 semester.

\section{PRELiminaries}

Throughout, we fix a prime $\mathrm{p} \geq 2$. The main figure to keep in mind is Figure 1 which illustrates the underlying graph of the quiver algebra $\mathrm{Z}$ in the cases $\mathrm{p}=3, \mathrm{p}=5$ and $\mathrm{p}=7$.

We also warn the reader that we will always have a $\rho$-shift of 1 and the crucial number will usually be "the highest weight plus 1 ", which we will denote by $v, w$ etc.

In the following, we review basic notation related to Z. For more details, we refer to [TW19].

Mathematics Subject Classification 2010. Primary: 20G05, 20C20; Secondary: 17B10.

Keywords. Modular representation theory, tilting modules, generators and relations, positive characteristic. 

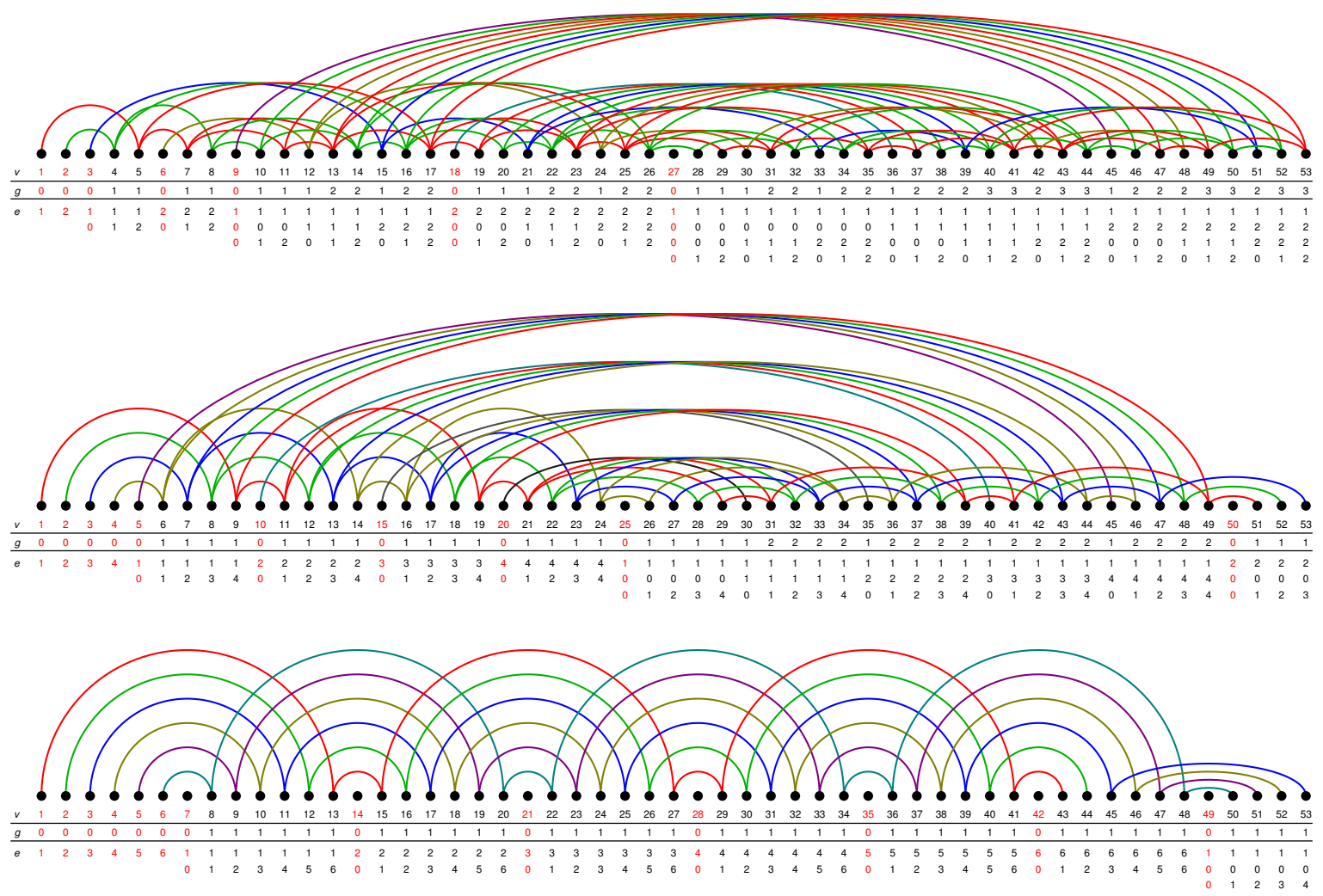

Figure 1. The full subquivers containing the first 53 vertices of the quiver underlying $\mathrm{Z}_{\mathrm{p}}$ for $\mathrm{p} \in\{3,5,7\}$, showing from top to bottom the numbers $v$ of the vertices, the generation of $v$ and the p-adic expansion of $v$.

\section{A. Some combinatorics of p-adic expansions.}

Definition 2.1 For any $v \in \mathbb{N}$ we write $\left[a_{j}, \ldots, a_{0}\right]_{\mathrm{p}}:=\sum_{i=0}^{j} a_{i} \mathrm{p}^{i}=v$ for the $\mathrm{p}$-adic expansion with digits $a_{i} \in\{0, \ldots, \mathrm{p}-1\}$ and $a_{j} \neq 0$. We sometimes also write $a_{h}$ for $h>j$, and then $a_{h}=0$, by convention. Finally, the generation of $v$ is the number of non-zero digits of $v$ minus 1 .

Remark 2.2 Conversely, we will sometimes specify numbers by p-adic expansions with negative digits such as $[3,-1,-6,-5,0,5,-6]_{7}=320048$.

The generation of $v$ is a complexity measure for the indecomposable tilting module of highest weight $v-1$. For example the indecomposables of generation zero are exactly the simple tilting modules. Moreover, to each non-simple indecomposable tilting module one associates another tilting module of generation one lower, called its mother, whose highest weight (plus 1) is obtained by setting the lowest non-zero digit to zero. Tracing the matrilinear ancestry of an indecomposable tilting module through decreasing generation numbers, one arrives at a simple tilting module, called an eve.

Definition 2.3 If $v=\left[a_{j}, \ldots, a_{0}\right]_{\mathrm{p}} \in \mathbb{N}$ is of generation zero, then $v$ is called an eve. The set of eves is denoted by Eve.

Note that $\mathrm{Eve}=\mathrm{Eve}^{<\mathrm{p}} \cup \mathrm{Eve}^{\geq \mathrm{p}}$, with $\mathrm{Eve}^{<\mathrm{p}}$ and Eve $\mathrm{e}^{\geq \mathrm{p}}$ having the evident meaning. 
Example 2.4 For $\mathrm{p}=7$ we have $\mathrm{Eve}^{<\mathrm{p}}=\{1,2,3,4,5,6\}$ and

$$
\mathrm{Eve}^{\geq \mathrm{p}}=\left\{[1,0]_{7},[2,0]_{7}, \ldots,[6,0]_{7},[1,0,0]_{7},[2,0,0]_{7}, \ldots,[6,0,0]_{7},[1,0,0,0]_{7}, \ldots\right\} .
$$

Definition 2.5 For two finite subsets $S, T \subset \mathbb{N}_{0}$ the distance between them is defined as $\mathrm{d}(S, T)=\min \{|s-t| \mid s \in S, t \in T\}$, and we write $T>S$ to indicate the requirement that every element in $T$ is strictly greater than every element in $S$.

Definition 2.6 For $S \subset \mathbb{N}_{0}$ a finite set, we consider partitions $S=\bigsqcup_{i} S_{i}$ of $S$ into subsets $S_{i}$ of consecutive integers, which we call stretches. We fix the coarsest such partition.

The set $S$ is called down-admissible for $v=\left[a_{j}, \ldots, a_{0}\right]_{\mathrm{p}}$ if:

(i) $a_{\min \left(S_{i}\right)} \neq 0$ for every $i$, and

(ii) if $s \in S$ and $a_{s+1}=0$, then $s+1 \in S$.

If $S \subset \mathbb{N}_{0}$ is down-admissible for $v=\left[a_{j}, \ldots, a_{0}\right]_{\mathrm{p}}$, then we define its downward reflection along $S$ as

$$
v[S]:=\left[a_{j}, \epsilon_{j-1} a_{j-1}, \ldots, \epsilon_{0} a_{0}\right]_{\mathrm{p}}, \quad \epsilon_{k}= \begin{cases}1 & \text { if } k \notin S, \\ -1 & \text { if } k \in S .\end{cases}
$$

Conversely, $S$ is up-admissible for $v=\left[a_{j}, \ldots, a_{0}\right]_{\mathrm{p}}$ if the following conditions are satisfied:

(i) $a_{\min \left(S_{i}\right)} \neq 0$ for every $i$, and

(ii) if $s \in S$ and $a_{s+1}=\mathrm{p}-1$, then we also have $s+1 \in S$.

If $S \subset \mathbb{N}_{0}$ is up-admissible for $v=\left[a_{j}, \ldots, a_{0}\right]_{\mathrm{p}}$, then we define its upward reflection along $S$ as

$$
v(S):=\left[a_{r(S)}^{\prime}, \ldots, a_{0}^{\prime}\right]_{\mathrm{p}}, \quad a_{k}^{\prime}= \begin{cases}a_{k} & \text { if } k \notin S, k-1 \notin S, \\ a_{k}+2 & \text { if } k \notin S, k-1 \in S, \\ -a_{k} & \text { if } k \in S,\end{cases}
$$

where we extend the digits of $v$ by $a_{h}=0$ for $h>j$ if necessary, and $r(S)$ is the biggest integer such that $a_{k}^{\prime} \neq 0$.

Example 2.7 Note that $S=\{7,6\}$ is up-admissible for $v=[3,1,6,5,0,5,6]_{7}$. To compute $v(S)$ we let $v=[0,3,1,6,5,0,5,6]_{7}$ and then we get

$$
v(S)=[0,3,1,6,5,0,5,6]_{7}=[2,-3,1,6,5,0,5,6]_{7}=[1,4,1,6,5,0,5,6]_{7} .
$$

The wave indicates the digits on which we apply $S$.

We tend to omit set brackets, e.g. for singleton sets $\{i\}$ we also write $v[i]$ and $v(i)$ instead of $v[\{i\}]$ and $v(\{i\})$.

Definition 2.8 If $S$ is up-admissible, then we denote by $\bar{S} \subset \mathbb{N}_{0}$ the down-admissible hull of $S$, the smallest down-admissible set containing $S$, if it exists.

Example 2.9 Let $\mathrm{p}=7$ and $v=[3,1,6,5,0,5,6]_{7}$.

(a) The singleton sets $\{2\},\{1\}$ and $\{i\}$ for $i \in \mathbb{N}_{>5}$ are not down-admissible for $v$. Of these only $\{1\}$ has a down-admissible hull and it is $\overline{\{1\}}=\{2,1\}$.

(b) Hence, the singleton sets $\{5\},\{4\},\{3\}$ and $\{0\}$ are minimal down-admissible for $v$, and we have

$$
v[5]=[3,-1,6,5,0,5,6]_{7}, \quad v[4]=[3,1,-6,5,0,5,6]_{7},
$$




$$
v[3]=[3,1,6,-5,0,5,6]_{7}, \quad v[0]=[3,1,6,5,0,5,-6]_{7} .
$$

Toghether with $\{2,1\}$, these are all minimal down-admissible sets for $v$.

(c) The singleton sets which are up-admissible for $v$ are $\{6\},\{5\},\{4\},\{1\}$ and $\{0\}$.

(d) The set $S=\{5,4,3 \mid 0\}$ is down- and up-admissible for $v$, and $v(S)$ and $v[S]$ can be illustrated via

$$
\begin{aligned}
v[5,4,3 \mid 0] & =[3, \underline{1,6,5}, 0,5,6]_{7}=[3,-1,-6,-5,0,5,-6]_{7}, \\
v(5,4,3 \mid 0) & =[3,1,6,5,0,5,6]_{7}=[5,-1,-6,-5,0,7,-6]_{7} .
\end{aligned}
$$

We have marked the digits where we reflect in the set $S$, either down or up.

2B. The Ringel dual. Let $\mathbb{K}=\overline{\mathbb{K}}$ denote a field of characteristic $\mathrm{p}$ with prime field $\mathbb{F}_{\mathrm{p}}$.

Definition 2.10 We define two functions $\mathrm{f}, \mathrm{g}: \mathbb{F}_{\mathrm{p}} \rightarrow \mathbb{F}_{\mathrm{p}}$ via

$$
\mathrm{f}(a)=\left\{\begin{array}{ll}
(-1)^{a} \frac{2}{a} & \text { if } 1 \leq a \leq \mathrm{p}-2, \\
0 & \text { if } a=0 \text { or } a=\mathrm{p}-1,
\end{array} \quad \mathrm{~g}(a)= \begin{cases}-\left(\frac{a+1}{a}\right) & \text { if } 1 \leq a \leq \mathrm{p}-1, \\
-2 & \text { if } a=0 .\end{cases}\right.
$$

Note that $\mathrm{f}(\mathrm{p}-1)=\mathrm{g}(\mathrm{p}-1)=0$ and $\mathrm{g}(a)=\mathrm{g}(\mathrm{p}-a-1)^{-1}$ for $a \neq 0, \mathrm{p}-1$.

Let Tilt denote the category of (left) tilting modules of $\mathrm{SL}_{2}(\mathbb{K})$, see e.g. [Wil17, Section 1] for a concise summary of the main definitions and properties regarding Tilt. Let $\mathrm{T}(v-1)$ denote (a choice of representative of) the indecomposable tilting module of highest weight $v-1$.

Definition 2.11 Define a $\mathbb{K}$-algebra

$$
\mathrm{Z}:=\bigoplus_{v, w \in \mathbb{N}} \operatorname{Hom}_{\text {Tilt }}(\mathrm{T}(v-1), \mathrm{T}(w-1)) .
$$

Let $\mathrm{e}_{v-1}$ be the idempotent in $\mathrm{Z}$ corresponding to $\mathrm{T}(v-1)$.

Definition 2.12 For each finite $S \subset \mathbb{N}_{0}$ we define scaling operators $\mathrm{f}_{S}, \mathrm{~g}_{S}, \mathrm{~h}_{S} \in \mathrm{Z}$ on $v=$ $\left[a_{j}, \ldots, a_{0}\right]_{\mathrm{p}}$ as

$$
\mathbf{f}_{S} \mathbf{e}_{v-1}=\mathrm{f}\left(a_{\max (S)+1}\right) \mathbf{e}_{v-1}, \quad \mathbf{g}_{S} \mathbf{e}_{v-1}=\mathrm{g}\left(a_{\max (S)+1}\right) \mathbf{e}_{v-1}, \quad \mathbf{h}_{S} \mathbf{e}_{v-1}=\mathrm{g}\left(a_{\max (S)+1}-1\right) \mathbf{e}_{v-1} .
$$

Example 2.13 Let again $\mathrm{p}=7, v=[3,1,6,5,0,5,6]_{7}$ and $S=\{5,4,3 \mid 0\}$. Then

$$
\mathbf{f}_{S} \mathbf{e}_{v-1}=\mathrm{f}(3) \mathbf{e}_{v-1}=4 \mathbf{e}_{v-1}, \quad \mathrm{~g}_{S} \mathbf{e}_{v-1}=\mathrm{g}(3) \mathbf{e}_{v-1}=\mathrm{e}_{v-1}, \quad \mathrm{~h}_{S} \mathbf{e}_{v-1}=\mathrm{g}(2) \mathbf{e}_{v-1}=2 \mathbf{e}_{v-1} .
$$

The following identifies $\mathrm{Z}$ explicitly, and can be taken as an abstract definition of a quiver algebra isomorphic to $\mathrm{Z}$, see also Remark 2.15 below.

Theorem 2.14 (See [TW19, Theorem 3.2].) The algebra $\mathrm{Z}$ is generated by $\mathrm{e}_{v-1}$ for $v \in \mathbb{N}$, and elements $\mathrm{D}_{S} \mathbf{e}_{v-1}$ and $\mathrm{U}_{S^{\prime}} \mathrm{e}_{v-1}$, where $S$ and $S^{\prime}$ denote minimal down-and up-admissible stretches for $v$, respectively. These generators are subject to the following complete set of relations.

(1) Idempotents.

$$
\mathrm{e}_{v-1} \mathrm{e}_{w-1}=\delta_{v, w} \mathrm{e}_{v-1}, \quad \mathbf{e}_{v[S]-1} \mathrm{D}_{S} \mathbf{e}_{v-1}=\mathrm{D}_{S} \mathrm{e}_{v-1}, \quad \mathbf{e}_{v\left(S^{\prime}\right)-1} \mathrm{U}_{S^{\prime}} \mathrm{e}_{v-1}=\mathrm{U}_{S^{\prime}} \mathrm{e}_{v-1} .
$$

(2) Containment. If $S^{\prime} \subset S$, then we have

$$
\mathrm{D}_{S^{\prime}} \mathrm{D}_{S} \mathrm{e}_{v-1}=0, \quad \mathrm{U}_{S} \mathrm{U}_{S^{\prime}} \mathrm{e}_{v-1}=0 .
$$

(3) Far-commutativity. If $\mathrm{d}\left(S, S^{\prime}\right)>1$, then

$$
\mathrm{D}_{S} \mathrm{D}_{S^{\prime}} \mathbf{e}_{v-1}=\mathrm{D}_{S^{\prime}} \mathrm{D}_{S} \mathbf{e}_{v-1}, \quad \mathrm{D}_{S} \mathrm{U}_{S^{\prime}} \mathbf{e}_{v-1}=\mathrm{U}_{S^{\prime}} \mathrm{D}_{S} \mathbf{e}_{v-1}, \quad \mathrm{U}_{S} \mathrm{U}_{S^{\prime}} \mathbf{e}_{v-1}=\mathrm{U}_{S^{\prime}} \mathrm{U}_{S} \mathbf{e}_{v-1} .
$$


(4) Adjacency relations. If $\mathrm{d}\left(S, S^{\prime}\right)=1$ and $S^{\prime}>S$, then

$$
\begin{array}{cl}
\mathrm{D}_{S^{\prime}} \mathrm{U}_{S} \mathbf{e}_{v-1}=\mathrm{D}_{S \cup S^{\prime}} \mathrm{e}_{v-1}, & \mathrm{D}_{S} \mathrm{U}_{S^{\prime}} \mathrm{e}_{v-1}=\mathrm{U}_{S^{\prime} \cup S} \mathbf{e}_{v-1}, \\
\mathrm{D}_{S^{\prime}} \mathrm{D}_{S} \mathbf{e}_{v-1}=\mathrm{U}_{S} \mathrm{D}_{S^{\prime}} \mathrm{h}_{S} \mathbf{e}_{v-1}, & \mathrm{U}_{S} \mathrm{U}_{S^{\prime}} \mathbf{e}_{v-1}=\mathrm{h}_{S} \mathrm{U}_{S^{\prime}} \mathrm{D}_{S} \mathbf{e}_{v-1} .
\end{array}
$$

(5) Overlap relations. If $S^{\prime} \geq S$ with $S^{\prime} \cap S=\{s\}$ and $S^{\prime} \not \subset S$, then we have

$$
\mathrm{D}_{S^{\prime}} \mathrm{D}_{S} \mathbf{e}_{v-1}=\mathrm{U}_{\{s\}} \mathrm{D}_{S} \mathrm{D}_{S^{\prime} \backslash\{s\}} \mathbf{e}_{v-1}, \quad \mathrm{U}_{S} \mathrm{U}_{S^{\prime}} \mathbf{e}_{v-1}=\mathrm{U}_{S^{\prime} \backslash\{s\}} \mathrm{U}_{S} \mathrm{D}_{\{s\}} \mathbf{e}_{v-1} .
$$

(6) Zigzag.

$$
\mathrm{D}_{S} \mathrm{U}_{S} \mathbf{e}_{v-1}=\mathrm{U}_{\bar{S}} \mathrm{D}_{\bar{S}} \mathbf{g}_{S} \mathbf{e}_{v-1}+\mathrm{U}_{T} \mathrm{U}_{\bar{S}} \mathrm{D}_{\bar{S}} \mathrm{D}_{T} \mathbf{f}_{S} \mathbf{e}_{v-1} .
$$

Here, if the down-admissible hull $\bar{S}$, or the smallest minimal down-admissible stretch $T$ with $T>\bar{S}$ does not exist, then the involved symbols are zero by definition.

The elements of the form

$$
\mathrm{e}_{w-1} \mathrm{U}_{S_{i_{l}}^{\prime}} \cdots \mathrm{U}_{S_{i_{0}}^{\prime}} \mathrm{D}_{S_{i_{0}}} \cdots \mathrm{D}_{S_{i_{k}}} \mathrm{e}_{v-1}
$$

with $S_{i_{l}}^{\prime}>\cdots>S_{i_{0}}^{\prime}$, and $S_{i_{0}}<\cdots<S_{i_{k}}$, form a basis for $\mathrm{e}_{w-1} \mathrm{Ze}_{v-1}$.

Finally, any word $\mathrm{e}_{w-1} \mathrm{Fe}_{v-1}$ in the generators of $\mathrm{Z}$ expands in (Basis) via (1)-(6).

Remark 2.15 In fact, as already mentioned above, we could alternatively define $\mathrm{Z}$ as the quiver algebra with underlying graphs as in Figure 1, using highest weights to index the vertices, and two arrows directed in opposite directions for each edge in this graph. The elements $\mathrm{e}_{v-1}$ are the vertex idempotents of this quiver algebra, and the elements $\mathrm{D}_{-}$, called down arrows, $\mathrm{U}_{-}$, called up arrows, are the arrows in this quiver, pointing left and right, respectively.

Remark 2.16 Note that all appearing scalars in the presentation of $\mathrm{Z}$ in Theorem 2.14 are from $\mathbb{F}_{\mathrm{p}}$ rather than $\mathbb{K}$.

Remark 2.17 In Theorem 2.14.(4) and (6), the right-hand sides of the shown relations feature morphisms indexed by admissible subsets that are not necessarily minimal. These morphisms are defined to be

$$
\mathrm{D}_{S} \mathrm{e}_{v-1}:=\mathrm{D}_{S_{i_{1}}} \cdots \mathrm{D}_{S_{i_{k}}} \mathrm{e}_{v-1}, \quad \mathrm{U}_{S^{\prime}} \mathrm{e}_{v-1}:=\mathrm{U}_{S_{i_{l}}^{\prime}} \cdots \mathrm{U}_{S_{i_{1}}^{\prime}} \mathrm{e}_{v-1},
$$

where the products are taken over the minimal down- respectively up-admissible stretches $S_{i_{j}}$ and $S_{i_{j}}^{\prime}$, such that $S=\bigsqcup_{j} S_{i_{j}}$ and $S^{\prime}=\bigsqcup_{j} S_{i_{j}}^{\prime}$, with $S_{i_{1}}<\cdots<S_{i_{k}}$ and $S_{i_{l}}^{\prime}>\cdots>S_{i_{1}}^{\prime}$.

Example 2.18 To be completely explicit with respect to Remark 2.15: if $\mathrm{p}=3$, then the quiver of the idempotent truncation of $\mathrm{Z}$ supported on the vertices $0,4,6,10,12$ and 16 would be

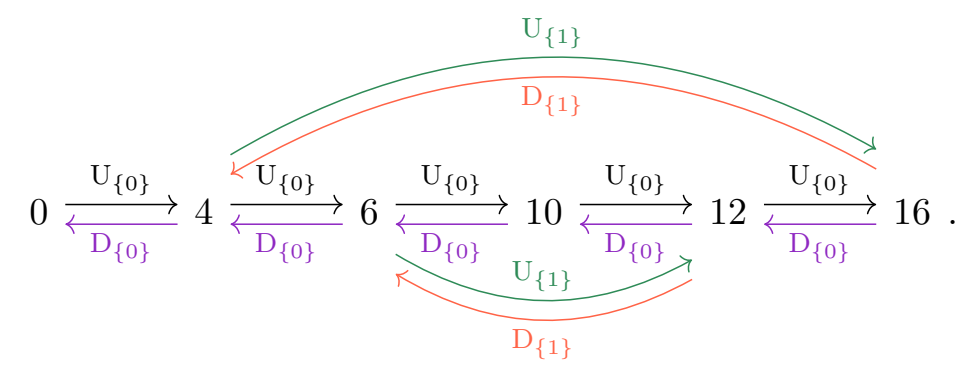

(The colors, here and below, are only to ease readability.) Here the labeling is coming from e.g.

$$
17=[1,2,2]_{3}, \quad 17[0]=[1,2, \underline{2}]_{3}=[1,2,-2]_{3}=13, \quad 17[1]=[1, \underline{2}, 2]_{3}=[1,-2,2]_{3}=5,
$$

which give the two downward arrows from 16, namely $\mathrm{D}_{\{0\}} \mathrm{e}_{16}=\mathrm{e}_{12} \mathrm{D}_{\{0\}}$ and $\mathrm{D}_{\{1\}} \mathrm{e}_{16}=\mathrm{e}_{4} \mathrm{D}_{\{1\}}$. We also have $\mathrm{D}_{\{1,0\}} \mathbf{e}_{16}=\mathrm{D}_{\{0\}} \mathrm{D}_{\{1\}} \mathbf{e}_{16}$. 
Definition 2.19 For any down-admissible set $S$ for $v$ we define the loop

$$
\mathrm{L}_{v-1}^{S}:=\mathrm{U}_{S} \mathrm{D}_{S} \mathrm{e}_{v-1} .
$$

Lemma 2.20 (See [TW19, Lemma 3.23].) Let $v \in \mathbb{N}$ with minimal down-admissible stretches $S_{j}, \ldots, S_{0}$. Then we have the $\mathbb{K}$-algebra isomorphism

$$
\operatorname{End}_{\text {Tilt }}(\mathrm{T}(v-1)) \cong \mathbb{K}\left[\mathrm{L}_{v-1}^{S_{j}}, \ldots, \mathrm{L}_{v-1}^{S_{0}}\right] /\left\langle\left(\mathrm{L}_{v-1}^{S_{j}}\right)^{2}, \ldots,\left(\mathrm{L}_{v-1}^{S_{0}}\right)^{2}\right\rangle
$$

and if $S$ is down-admissible for $v$, then $\mathrm{L}_{v-1}^{S}=\prod_{k \mid S_{k} \subset S} \mathrm{~L}_{v-1}^{S_{k}}$.

Example 2.21 Again, let us consider $\mathrm{p}=7$ and $v=[3,1,6,5,0,5,6]_{7}$. Recall that we have calculated the minimal down-admissible stretches of $v$ in Example 2.9.(b). Hence, $\operatorname{End}_{\mathbf{T i l t}}(\mathrm{T}(v-1))$ has generators $\mathrm{L}_{v-1}^{\{5\}}, \mathrm{L}_{v-1}^{\{4\}}, \mathrm{L}_{v-1}^{\{3\}}$ and $\mathrm{L}_{v-1}^{\{0\}}$ as well as $\mathrm{L}_{v-1}^{\{2,1\}}$. The maximal loop $\mathrm{L}_{v-1}^{\{5|4| 3|2,1| 0\}}=$ $\mathrm{L}_{v-1}^{\{5\}} \mathrm{L}_{v-1}^{\{4\}} \mathrm{L}_{v-1}^{\{3\}} \mathrm{L}_{v-1}^{\{2,1\}} \mathrm{L}_{v-1}^{\{0\}}$ can be thought of as a head-to-socle map on $\mathrm{T}(v-1)$.

For later use we also recall:

Lemma 2.22 (See [TW19, Lemma 3.23].) We have

$$
\mathrm{D}_{S} \mathrm{U}_{S} \mathrm{D}_{S} \mathrm{e}_{v-1}=0, \quad \mathrm{e}_{v-1} \mathrm{U}_{S} \mathrm{D}_{S} \mathrm{U}_{S}=0,
$$

whenever $S$ is down-admissible for $v$.

2C. Closures of the algebra. Using Theorem 2.14 we get a sequence

$$
\mathrm{z}^{1} \nleftarrow \mathrm{Z}^{2} \leftarrow \mathrm{z}^{3} \nleftarrow \ldots \leftarrow \lim _{\longleftarrow} \mathrm{Z}^{i}=: \overline{\mathrm{z}},
$$

where $\mathrm{Z}^{i}$ is the quotient of $\mathrm{Z}$ obtained by the ideal generated by $\left\{\mathbf{e}_{v-1} \mid v>i\right\}$. (Note that the category of $\mathbb{K}$-algebras is complete, i.e. has all limits, so $\bar{Z}$ is indeed a $\mathbb{K}$-algebra.

Remark 2.23 Note that elements in $\mathrm{Z}$ are finite $\mathbb{K}$-linear combinations of elements from Theorem 2.14.(Basis), while elements in $\overline{\mathrm{Z}}$ can be (countably) infinite $\mathbb{K}$-linear combinations of these. In particular, the unit of $\overline{\mathrm{Z}}$ is

$$
1=\sum_{v \in \mathbb{N}} \mathbf{e}_{v-1} \in \bar{Z}
$$

while $\mathrm{Z}$ is only locally unital.

Below $\mathrm{Z}^{\prime}$ will denote either $\mathrm{Z}$ or $\overline{\mathrm{Z}}$.

\section{THE CENTER OF THE QUIVER ALGEBRA}

We will now compute the center of $Z^{\prime}$.

3A. Reduction to connected components. Note that Theorem 2.14 implies that the $\mathbb{K}$ algebra $\overline{\mathrm{Z}}$ decomposes as

$$
\begin{aligned}
& \mathrm{Z}=\bigoplus_{e \in \operatorname{Eve}} \mathrm{Z}_{e-1}, \quad \mathrm{Z}_{e-1}:=\bigoplus_{v, w \in(e)_{\mathrm{p}}} e_{w-1} \mathrm{Z} e_{v-1}, \\
& \overline{\mathrm{Z}}=\prod_{e \in \operatorname{Eve}} \overline{\mathrm{Z}}_{e-1}, \quad \overline{\mathrm{Z}}_{e-1}:=\prod_{v, w \in(e)_{\mathrm{p}}} e_{w-1} \overline{\mathrm{Z}} e_{v-1} .
\end{aligned}
$$

Here $(e)_{\mathrm{p}}$ denotes the set of natural numbers $v$ such that the vertex $v-1$ is in the connected component of the graph underlying $\mathrm{Z}$ which contains $e-1$.

Example 3.1 As can be seen in Figure $1,(1)_{3}=\{1,5,7,11,13,17, \ldots\}$, see also Example 2.18.

Letting $Z\left({ }_{-}\right)$denote the center of an algebra, the following is thus immediate. 
Lemma 3.2 We have $Z(\mathrm{Z})=\bigoplus_{e \in \mathrm{Eve}} Z\left(\mathrm{Z}_{e-1}\right)$ and $Z(\overline{\mathrm{Z}})=\prod_{e \in \mathrm{Eve}} Z\left(\overline{\mathrm{Z}}_{e-1}\right)$

The presentation of $\mathrm{Z}^{\prime}$ also immediately gives:

Lemma 3.3 (See [TW19, Proposition 5.3].) There are isomorphisms of algebras $\mathrm{Z}_{e-1}^{\prime} \cong \mathrm{Z}_{e^{\prime}-1}^{\prime}$ for all $e, e^{\prime} \in$ Eve with equal non-zero digits.

Remark 3.4 In fact $\mathrm{Z}_{e-1}^{\prime} \cong \mathrm{Z}_{e^{\prime}-1}^{\prime}$ for all $e, e^{\prime} \in$ Eve, regardless of the digits. But the isomorphism is, in contrast to the one from Lemma 3.3, not immediate from Theorem $A$, the reason being the scalars $\mathrm{f}_{S}, \mathrm{~g}_{S}$ and $\mathrm{h}_{S}$ appearing therein.

Thus, it suffices to compute $Z\left(\mathrm{Z}_{e-1}^{\prime}\right)$ for $e \in \mathrm{Eve}^{<\mathrm{p}} \subset$ Eve.

\section{B. The central elements.}

Definition 3.5 Let $v=\left[a_{j}, \ldots, a_{0}\right]_{\mathrm{p}} \in \mathbb{N}$. For $i \neq j$ and $a_{i} \neq 0$, we define elements in $\mathrm{Z}^{\prime}$ by

$$
\mathrm{D}_{i} \mathrm{e}_{v-1}:=\mathrm{D}_{\overline{\{i\}}} \mathrm{e}_{v-1}, \quad \mathrm{e}_{v-1} \mathrm{U}_{i}:=\mathrm{e}_{v-1} \mathrm{U}_{\overline{\{i\}}}, \quad \mathrm{L}_{i} \mathrm{e}_{v-1}:=(-1)^{a_{i}} a_{i} \mathrm{U}_{i} \mathrm{D}_{i} \mathrm{e}_{v-1} .
$$

Furthermore, we define

$$
\mathrm{D}(v):=\left\{i \in \mathbb{N}_{0} \mid i<j, a_{i} \neq 0\right\} .
$$

In words, $\mathrm{D}(v)$ is the set of non-zero, non-leading digits of $v=\left[a_{j}, \ldots, a_{0}\right]_{\mathrm{p}}$. For $\mathrm{S} \subset \mathrm{D}(v)$ we consider

$$
\mathrm{L}_{\mathrm{S}} \mathrm{e}_{v-1}:=\prod_{i \in \mathrm{S}} \mathrm{L}_{i} \mathrm{e}_{v-1}
$$

Note that the factors in (3-1) commute by Lemma 2.20 .

Example 3.6 For $\mathrm{p}=7$ and $v=[3,1,6,5,0,5,6]_{7}$ we have $\mathrm{D}(v)=\{0,1,3,4,5\}$. Thus, for $\mathrm{S}=\mathrm{D}(v)$ we have $\mathrm{L}_{\mathrm{S}} \mathrm{e}_{v-1}=\mathrm{L}_{5} \mathrm{~L}_{4} \mathrm{~L}_{3} \mathrm{~L}_{1} \mathrm{~L}_{0} \mathrm{e}_{v-1}$.

Definition 3.7 Let $\mathrm{S} \subset \mathbb{N}_{0}$ be finite. We define an equivalence relation $\sim_{\mathrm{S}}$ on $\mathbb{N}$ as follows. First, we let $v \sim_{\mathrm{S}} v$ for all $v \in \mathbb{N}$. Further, for $v, w \in \mathbb{N}$ with $v \neq w$ we declare $v \sim_{\mathrm{S}} w$ if $\mathrm{S} \subset \mathrm{D}(v) \cap \mathrm{D}(w)$ and $v=w[k]$ or $w=v[k]$ for some $k \notin \mathrm{S}$, Finally, we take the transitive closure.

In words, we have $v \sim_{\mathrm{S}} w$ if and only if either $v=w$, or $v-1$ and $w-1$ are connected by a chain of arrows $\mathrm{D}_{k}$ or $\mathrm{U}_{k}$ for $k \notin \mathrm{S}$ such that all vertices that are involved have $\mathrm{S}$ among their non-maximal and non-zero digits. We call such a chain an S-path.

Definition 3.8 For $v \in(e)_{\mathrm{p}}$ we introduce notation for the $\sim_{\mathrm{D}(v)}$ equivalence class of $v$ :

$$
(v)_{\mathrm{p}}^{\odot}:=\left\{w \in(e)_{\mathrm{p}} \mid v \sim_{\mathrm{D}(v)} w\right\} .
$$

Example 3.9 Take again $\mathrm{p}=7, v=[3,1,6,5,0,5,6]_{7}$ and $\mathrm{S}=\mathrm{D}(v)=\{0,1,3,4,5\}$. Then:

(a) We have $w=v(5,4,3)=[4,5,0,2,0,5,6]_{7} \notin(v)_{\mathrm{p}}^{\odot}$, since $4 \in \mathrm{D}(v)$, but $4 \notin \mathrm{D}(w)$.

(b) Recall that the only up-admissible singleton set not in $\mathrm{D}(v)$ is $\{6\}$, see Example 2.9.(c). Thus, the only direct neighbor of $v$ in $(v)_{\mathrm{p}}^{\odot}$ is $v_{1}=v(6)=[1,4,1,6,5,0,5,6]_{7}$.

(c) The set $(v)_{\mathrm{p}}^{\odot}$ is an infinite set since $v_{1}=v(6)=[1,4,1,6,5,0,5,6]_{7} \in(v)_{\mathrm{p}}^{\odot}$, and recursively $v_{k}=v_{k-1}(5+k) \in(v)_{\mathrm{p}}^{\odot}$. Note that the first six digits of the $v_{k}$ agree with those of $v$.

Lemma 3.10 Let $v=\left[a_{j}, \ldots, a_{0}\right]_{\mathrm{p}} \in \mathbb{N}$ and $w=\left[b_{k}, \ldots, b_{0}\right]_{\mathrm{p}} \in \mathbb{N}$. If $w \in(v)_{\mathrm{p}}^{\odot}$, then $b_{i}=a_{i}$ for $1 \leq i<j$. 
Proof. We prove by induction on the length $\ell$ of a $\mathrm{D}(v)$-path that the first $j$ digits of all elements $w \in(v)_{\mathrm{p}}^{\odot}$ agree with those of $v$. For $\ell=0$ there is nothing to prove. Now suppose $w$ is at the end of a $\mathrm{D}(v)$-path of length $\ell \geq 1$, whose last step is a morphism $\mathrm{U}_{k}$ or $\mathrm{D}_{k}$ from $w^{\prime}$ to $w$, with $k \notin \mathrm{D}(v)$. Since $w^{\prime}$ is reached from $v$ by a $\mathrm{D}(v)$-path of length $\ell-1$, the induction hypothesis implies that the first $j$ digits of $w^{\prime}$ agree with those of $v$. In particular, each of the first $j$ digits is either in $\mathrm{D}(v)$ or zero, and so $k \geq j$. Then $w=w^{\prime}[k]$, respectively $w=w^{\prime}(k)$, have the same first $j$ digits as $w^{\prime}$ and also as $v$.

Lemma 3.10 implies that $(v)_{\mathrm{p}}^{\odot}$ for $v=\left[a_{j}, \ldots, a_{0}\right]_{\mathrm{p}} \in \mathbb{N}$ is a shifted copy of the block $\left(a_{j}\right)_{\mathrm{p}}$, see [TW19, Proposition 5.2]. As a consequence we get the following.

Corollary 3.11 For every $v \in \mathbb{N}$, the set $(v)_{\mathbf{p}}^{\odot}$ is infinite, and if $w \in(v)_{\mathbf{p}}^{\odot}$, then $w \geq v$.

The loops that we define now are only elements in $\bar{Z}$ but not in $\mathrm{Z}$.

Definition 3.12 For $v \in(e)_{\mathrm{p}}$ and $v \neq e$ we define elements

$$
\mathrm{L}_{v}:=\sum_{w \in(v)_{\mathrm{p}}^{\odot}} \mathrm{L}_{\mathrm{D}(v)} \mathrm{e}_{w-1} \in \overline{\mathrm{Z}}_{e-1} .
$$

In words, $\mathrm{L}_{v}$ consists of the maximal loop at $v-1$, together with the sum of all loops of the same type on $w-1$ for all $w$ in the shifted block $(v)_{\mathbf{p}}^{\odot}$.

3C. The center. By Lemma 3.2, the following computes the center $Z\left(\mathrm{Z}^{\prime}\right)$ :

Theorem 3.13 For $e \in$ Eve $^{<\mathrm{p}}$ we have algebra isomorphisms

$$
Z\left(\mathrm{Z}_{e-1}\right) \cong\{0\}, \quad Z\left(\overline{\mathrm{Z}}_{e-1}\right) \stackrel{\cong}{\rightrightarrows} \mathbb{K}\left[X_{v} \mid v \in(e)_{\mathrm{p}} \backslash\{e\}\right] /\left\langle X_{v} X_{w} \mid v, w \in(e)_{\mathrm{p}} \backslash\{e\}\right\rangle, \quad \mathrm{L}_{v} \mapsto X_{v} .
$$

Proof. We first observe that we have $Z\left(\mathrm{Z}_{e-1}^{\prime}\right) \subset \prod_{v \in(e)_{\mathrm{p}}} \mathrm{e}_{v-1} \mathrm{Z}^{\prime} \mathrm{e}_{v-1}$. Indeed, if $z \in Z\left(\mathrm{Z}_{e-1}^{\prime}\right)$ and $v \in(e)_{\mathrm{p}}$, then we have $z \mathrm{e}_{v-1}=z \mathrm{e}_{v-1}^{2}=\mathrm{e}_{v-1} z \mathrm{e}_{v-1}$. The observation follows since $z$ is by assumption a finite or infinite sum of terms $z \mathbf{e}_{v-1}$ for $v \in(e)_{\mathbf{p}}$.

- Let us first consider $\bar{Z}_{e-1}$. Here Lemma 3.17.(b) (proven below) shows that the infinite sums $\mathrm{L}_{v}$ defined above are in the center $Z\left(\bar{Z}_{e-1}\right)$, while Lemma 3.19 (also proven below) shows that they, together with the identity, give a basis of $Z\left(\bar{Z}_{e-1}\right)$. Thus,

$$
Z\left(\overline{\mathrm{Z}}_{e-1}\right) \cong \mathbb{K}\left\langle 1, \mathrm{~L}_{v} \mid v \in(e)_{\mathrm{p}} \backslash\{e\}\right\rangle .
$$

The second isomorphism then follows because we already know the relations among the $\mathrm{L}_{v}$, cf. Lemma 3.15 .

- For the first isomorphism, note that we have an inclusion $Z\left(\mathrm{Z}_{e-1}\right) \hookrightarrow Z\left(\overline{\mathrm{Z}}_{e-1}\right)$ of non-unital $\mathbb{K}$-algebras. However, Lemma 3.17 implies that all non-trivial elements of $Z\left(\bar{Z}_{e-1}\right)$ are supported on infinitely many idempotents, so $Z\left(\mathrm{Z}_{e-1}\right)=Z\left(\bar{Z}_{e-1}\right) \cap \mathrm{Z}_{e-1}=\{0\}$.

3D. Some lemmas for the proof of Theorem 3.13. Next, we identify the relations among the $\mathrm{L}_{v}$.

Lemma 3.14 For $e \in \mathrm{Eve}^{<\mathrm{p}}$ and $v \in(e)_{\mathrm{p}}$ with $v \neq e$ we have $0 \in \mathrm{D}(v)$.

Proof. Any $v \in(e)_{\mathrm{p}}$ can be reached from $e$ through a finite sequence of $\mathrm{D}_{i}$ and $\mathrm{U}_{i}$. It is straightforward to check that under each such an arrow the zeroth digit stays unchanged or is reflected to its negative. The zeroth digit of any $v$ is, thus, either $e$ or $\mathrm{p}-e$. Unless $v=e$, this implies $0 \in \mathrm{D}(v)$.

Lemma 3.15 For $e \in \mathrm{Eve}^{<\mathrm{p}}$ and $v, w \in(e)_{\mathrm{p}} \backslash\{e\}$ we have $\mathrm{L}_{v} \mathrm{~L}_{w}=0$. 
Proof. By Lemma 3.14 we have $0 \in \mathrm{D}(v)$ for any $v \in(e)_{\mathrm{p}}$ with $v \neq e$. If $(v)_{\mathrm{p}}^{\odot} \cap(w)_{\mathrm{p}}^{\odot}=\emptyset$, then we have $\mathrm{L}_{v} \mathrm{~L}_{w}=0$ trivially. Otherwise the product $\mathrm{L}_{v} \mathrm{~L}_{w}$ is supported on certain $z \in(v)_{\mathrm{p}}^{\odot} \cap(w)_{\mathrm{p}}^{\odot}$, but there it is a multiple of $\mathrm{L}_{0}^{2} \mathrm{e}_{w-1}=0$, see Lemma 2.20.

Next will be that the $\mathrm{L}_{v}$ are central, which needs:

Lemma 3.16 Let $v \in(e)_{\mathrm{p}}$ and $i, k \in \mathrm{D}(v)$ with $i \neq k$ and $i \in \mathrm{D}(v[k])$. Then we have:

$$
\begin{aligned}
\mathrm{e}_{v-1} \mathrm{~L}_{i} \mathrm{U}_{k} \mathrm{e}_{v[k]-1} & =\mathrm{e}_{v-1} \mathrm{U}_{k} \mathrm{~L}_{i} \mathrm{e}_{v[k]-1} \neq 0, \\
\mathrm{e}_{v-1} \mathrm{~L}_{i} \mathrm{U}_{i} \mathrm{e}_{v[i]-1} & =\mathrm{e}_{v-1} \mathrm{U}_{i} \mathrm{~L}_{i} \mathrm{e}_{v[i]-1}=0 .
\end{aligned}
$$

In particular, a loop of type $\mathrm{L}_{i}$ can be transported along $\mathrm{U}_{k}$, and thus, also along $\mathrm{D}_{k}$, due to symmetry.

Proof. We rewrite both sides of the desired equations and introduce notation as follows. Here we allow the case $k=i$, for which we have to prove that both sides are zero.

$$
\begin{aligned}
& \mathrm{L}_{i} \mathrm{U}_{k} \mathrm{e}_{v[k]-1}=(-1)^{a_{i}} a_{i} \mathrm{U}_{S_{i}} \mathrm{D}_{S_{i}} \mathrm{U}_{S_{k}} \mathrm{e}_{v[k]-1} \\
& \mathrm{U}_{k} \mathrm{~L}_{i} \mathrm{e}_{v[k]-1}=(-1)^{b_{i}} b_{i} \mathrm{U}_{S_{k}} \mathrm{U}_{T_{i}} \mathrm{D}_{T_{i}} \mathrm{e}_{v[k]-1},
\end{aligned}
$$

where $S_{i}=\overline{\{i\}}$ and $S_{k}=\overline{\{k\}}$ are the down-admissible hulls for $v, T_{i}:=\overline{\{i\}}$ is the down-admissible hull for $v[k]$, and $b_{i}$ denotes the $i$ th digit of $v[k]$. In checking (3-3) and (3-4), there are four cases to consider.

- If $S_{i}$ and $S_{k}$ are distant, then $T_{i}=S_{i}$ and $a_{i}=b_{i}$, and (3-3) follows from far-commutativity Theorem 2.14.(3).

- If $S_{k}>S_{i}$ are adjacent, then we have $a_{i}=b_{i}$ and $S_{i}$ is down-admissible for $v[k]$, which implies $S_{i}=T_{i}$. Now we distinguish two cases. Suppose $a_{k} \neq 1$, then we compute

$$
\begin{aligned}
\mathrm{U}_{S_{i}} \mathrm{D}_{S_{i}} \mathrm{U}_{S_{k}} \mathrm{e}_{v[k]-1} & =\mathrm{U}_{S_{i}} \mathrm{U}_{S_{k}} \mathrm{U}_{S_{i}} \mathrm{e}_{v[k]-1} \\
& =\mathrm{g}\left(a_{k}-1\right) \mathrm{U}_{S_{k}} \mathrm{D}_{S_{i}} \mathrm{U}_{S_{i}} \mathrm{e}_{v[k]-1} \\
& =\mathrm{g}\left(a_{k}-1\right) \mathrm{U}_{S_{k}}\left(\mathrm{~g}\left(\mathrm{p}-a_{k}\right) \mathrm{U}_{S_{i}} \mathrm{D}_{S_{i}}+\mathrm{f}\left(\mathrm{p}-a_{k}\right) \mathrm{U}_{S_{k}} \mathrm{U}_{S_{i}} \mathrm{D}_{S_{i}} \mathrm{D}_{S_{k}}\right) \mathrm{e}_{v[k]-1} \\
& =\mathrm{U}_{S_{k}} \mathrm{U}_{S_{i}} \mathrm{D}_{S_{i}} \mathrm{e}_{v[k]-1}=\mathrm{U}_{S_{k}} \mathrm{U}_{T_{i}} \mathrm{D}_{T_{i}} \mathrm{e}_{v[k]-1},
\end{aligned}
$$

as the $\mathrm{f}$ term gets killed by containment Theorem 2.14.(2) and we have $\mathrm{g}\left(a_{k}-1\right) \mathrm{g}\left(\mathrm{p}-a_{k}\right)=$ 1 , because $a_{k} \neq 1$.

Now suppose $a_{k}=1$. Then we compute:

$$
\begin{aligned}
\mathrm{U}_{S_{i}} \mathrm{D}_{S_{i}} \mathrm{U}_{S_{k}} \mathrm{e}_{v[k]-1} & =\mathrm{U}_{S_{i}} \mathrm{U}_{S_{k} \cup\{k-1\}} \mathrm{U}_{S_{i} \backslash\{k-1\}} \mathrm{e}_{v[k]-1} \\
& =\mathrm{U}_{S_{k}} \mathrm{U}_{S_{i}} \mathrm{D}_{\{k-1\}} \mathrm{U}_{S_{i} \backslash\{k-1\}} \mathrm{e}_{v[k]-1} \\
& =\mathrm{U}_{S_{k}} \mathrm{U}_{S_{i}} \mathrm{D}_{S_{i}} \mathrm{e}_{v[k]-1},
\end{aligned}
$$

where we have used the overlap relation Theorem 2.14.(5). In either case, we deduce (3-3) since $a_{i}=b_{i}$.

- Suppose that $S_{i}>S_{k}$ are adjacent. Then we have $a_{i}-1=b_{i}$. By the admissibility assumption we have $b_{i} \neq 0$, which implies $T_{i}=S_{i}$, and we compute

$$
\begin{aligned}
\mathrm{U}_{S_{k}} \mathrm{U}_{T_{i}} \mathrm{D}_{T_{i}} \mathrm{e}_{v[k]-1} & =\mathrm{U}_{S_{k}} \mathrm{U}_{S_{i}} \mathrm{D}_{S_{i}} \mathrm{e}_{v[k]-1} \\
& =\mathrm{g}\left(a_{i}-1\right) \mathrm{U}_{S_{i}} \mathrm{D}_{S_{k}} \mathrm{D}_{S_{i}} \mathrm{e}_{v[k]-1} \\
& =\mathrm{g}\left(a_{i}-1\right) \mathrm{U}_{S_{i}} \mathrm{D}_{S_{i}} \mathrm{U}_{S_{k}} \mathrm{e}_{v[k]-1} .
\end{aligned}
$$

Here we observe $\mathrm{g}\left(a_{i}-1\right)=-\frac{a_{i}}{a_{i}-1}=\frac{(-1)^{a_{i}} a_{i}}{(-1)^{b_{i} b_{i}}}$, which verifies (3-3). 
- Finally, if $S_{i}=S_{k}$, then we have $\mathrm{U}_{S_{i}} \mathrm{D}_{S_{i}} \mathrm{U}_{S_{i}} \mathrm{e}_{v[k]-1}=0$ by (2-2). For the other side of the equation we consider $\mathrm{U}_{S_{i}} \mathrm{U}_{T_{i}} \mathrm{D}_{T_{i}} \mathrm{e}_{v[k]-1}$. If $T_{i}=\{i\}$, then $S_{i} \supset T_{i}$ and the expression is zero by the containment relation Theorem 2.14.(2). Otherwise we necessarily have $S_{i}=\{i\} \subset T_{i}$ and we use the overlap relation Theorem 2.14.(5) to get

$$
\mathrm{U}_{S_{i}} \mathrm{U}_{T_{i}} \mathrm{D}_{T_{i}} \mathrm{e}_{v[k]-1}=\mathrm{U}_{T_{i} \backslash S_{i}} \mathrm{U}_{S_{i}} \mathrm{D}_{S_{i}} \mathrm{D}_{T_{i}} \mathrm{e}_{v[k]-1}=0,
$$

where we have again used containment at the end. Hence, (3-4) holds.

Lemma 3.17 We have the following:

(a) If $z \in Z\left(\overline{\mathrm{Z}}_{e-1}\right)$ satisfies $z \mathrm{e}_{v-1}=c \mathrm{~L}_{v} \mathbf{e}_{v-1}$ for some $v \in(e)_{\mathbf{p}} \backslash\{e\}$ and $c \in \mathbb{K}$, then $z \mathrm{e}_{w-1}$ also contains $\mathrm{L}_{v} \mathrm{e}_{w-1}$ with coefficient $c$ for any $w \in(v)_{\mathrm{p}}^{\odot}$.

(b) For $v \in(e)_{\mathrm{p}} \backslash\{e\}$ we have $\mathrm{L}_{v} \in Z\left(\overline{\mathrm{Z}}_{e-1}\right)$.

Proof. For the first part, expand $\mathrm{L}_{v}$ into a product of basic loops and observe that the $w \in(v)_{\mathrm{p}}^{\odot}$ are precisely the vertices to which $\mathrm{L}_{v}$ can be transported using Lemma 3.16. In a central element, these loops $\mathrm{L}_{v}$ therefore have to appear with the same coefficient at any $w \in(v)_{\mathrm{p}}^{\odot}$.

For the second part, we write $v=\left[a_{j}, \ldots, a_{0}\right]_{\mathrm{p}}$ and observe that every arrow $\mathrm{U}_{k}$ or $\mathrm{D}_{k}$ in the quiver, for which we write $Y_{k}$, is one of the following:

(i) $Y_{k}$ is not adjacent to any $w \in(v)_{\mathrm{p}}^{\odot}$, in which case it trivially commutes:

$$
Y_{k} \mathrm{~L}_{v}=0, \quad \mathrm{~L}_{v} Y_{k}=0 .
$$

(ii) $Y_{k}$ is adjacent to an $w \in(v)_{\mathrm{p}}^{\odot}$, but not a generator for the equivalence relation $\sim_{\mathrm{D}(v)}$. In this case Lemma 3.10 implies $k \leq j$ and commutation follows from (3-4).

(iii) $Y_{k}$ is a generating arrow of the equivalence relation $\sim_{\mathrm{D}(v)}$, in which case it commutes with the loops of type $\mathrm{L}_{\mathrm{D}(v)}$ by (3-3).

The following example shows that the assumption $i \in \mathrm{D}(v[k])$ in Lemma 3.16 is necessary.

Example 3.18 Let $\mathrm{p}=3$. Then there is no element in $z \in Z\left(\bar{Z}_{0}\right)$ such that $z \mathrm{e}_{12}=\mathrm{U}_{1} \mathrm{D}_{1} \mathrm{e}_{12}$. Indeed, we can compute

$$
z \mathrm{e}_{10} \mathrm{D}_{0} \mathrm{e}_{12}=\mathrm{D}_{0} z \mathrm{e}_{12}=\mathrm{D}_{0} \mathrm{U}_{1} \mathrm{D}_{1} \mathrm{e}_{12}=\mathrm{U}_{1,0} \mathrm{D}_{1} \mathrm{e}_{12}
$$

However, $\mathrm{U}_{1,0} \mathrm{D}_{1} \mathrm{e}_{12} \neq z \mathrm{e}_{10} \mathrm{D}_{0} \mathrm{e}_{12}$ for any $z \mathrm{e}_{10}$, as is easily verified since the endomorphisms of $\mathrm{e}_{10}$ are spanned by $\mathrm{e}_{10}$ and $\mathrm{U}_{0} \mathrm{D}_{0} \mathrm{e}_{10}$.

Neither is there $z \in Z\left(\bar{Z}_{0}\right)$ such that $z \mathrm{e}_{16}=\mathrm{U}_{1} \mathrm{D}_{1} \mathrm{e}_{16}$, as such a loop, if central, could be transported to $\mathrm{e}_{12}$.

Finally, we show that $\mathrm{L}_{v}$, together with the unit, form a basis of the center.

Lemma 3.19 The center $Z\left(\overline{\mathrm{Z}}_{e-1}\right)$ has a basis given by the unit (cf. (2-3)) and the $\mathrm{L}_{v}$ for $v \in(e)_{\mathrm{p}}$ with $v \neq e$.

Proof. To see linear independence, first note that no linear combination of the (nilpotent) $\mathrm{L}_{v}$ can be the unit. Moreover, assuming that $\sum_{i=1}^{r} a_{i} \mathrm{~L}_{v_{i}}=0$ for $a_{i} \in \mathbb{K}$ and $v_{i}<v_{j}$ for $i<j$, we can multiply this equation with $\mathrm{e}_{v_{1}-1}$ and get $a_{1} \mathrm{~L}_{\mathrm{D}\left(v_{1}\right)} \mathrm{e}_{v_{1}-1}=a_{1} \mathrm{~L}_{v_{1}} \mathrm{e}_{v_{1}-1}=\sum_{i=1}^{r} a_{i} \mathrm{~L}_{v_{i}} \mathrm{e}_{v_{1}-1}=0$. Hence, $a_{1}=0$, since $\mathrm{L}_{\mathrm{D}\left(v_{1}\right)} \mathrm{e}_{v_{1}-1}$ is a basis element in the corresponding endomorphism ring. We can repeat this process to show that all $a_{i}$ are zero.

Suppose now we are given $z \in Z\left(\overline{\mathrm{Z}}_{e-1}\right)$. Let $v \in(e)_{\mathrm{p}}$ be minimal with $z \mathrm{e}_{v-1} \neq 0$. If $v=e$, then $z \mathbf{e}_{v-1}=c \cdot \mathbf{e}_{v-1}$ for some non-zero scalar $c \in \mathbb{K}$. In this case, we proceed with the central element $z-c \cdot 1$ in place of $z$. 
Now we assume that $v \neq e$ and claim that $z \mathbf{e}_{v-1}=c \cdot \mathrm{L}_{v} \mathbf{e}_{v-1}$ for some non-zero scalar $c \in \mathbb{K}$ since otherwise we could find a smaller $v$.

To see this, suppose that $z \mathbf{e}_{v-1}$ contains a summand $d \cdot \mathrm{L}_{S} \mathbf{e}_{v-1}$ and suppose there is an $i \in \mathrm{D}(v) \backslash S$. We write $S_{i}:=\overline{\{i\}}$ for the admissible hull and split $S=S_{>i} \cup S_{<i}$ into subsets of indices greater and smaller than $i$ respectively. Then $z \mathrm{D}_{i} \mathrm{e}_{v-1}=\mathrm{D}_{i} z \mathrm{e}_{v-1}$ contains a summand

$$
\begin{aligned}
d \cdot \mathrm{D}_{S_{i}} \mathrm{~L}_{S} \mathrm{e}_{v-1} & =d \cdot \mathrm{D}_{S_{i}} \mathrm{U}_{S_{>i}} \mathrm{U}_{S_{<i}} \mathrm{D}_{S_{<i}} \mathrm{D}_{S_{>i}} \mathrm{e}_{v-1} \\
& = \begin{cases}d \cdot \mathrm{U}_{S_{>i} \cup S_{i}} \mathrm{U}_{S_{<i}} \mathrm{D}_{S_{<i}} \mathrm{D}_{S_{>i}} \mathrm{e}_{v-1} & \text { if } S_{>i} \text { is adjacent to } i, \\
d \cdot \mathrm{U}_{S_{>i}} \mathrm{U}_{S_{<i}} \mathrm{D}_{S_{<i}} \mathrm{D}_{i} \mathrm{D}_{S_{>i}} \mathrm{e}_{v-1} & \text { if } S_{>i} \text { is distant to } i,\end{cases}
\end{aligned}
$$

where we have used the adjacency relation Theorem 2.14.(4) in the first case, the far-commutativity relation Theorem 2.14.(3) in the second case, and also that $\mathrm{D}_{S_{i}}$ commutes with $\mathrm{U}_{S_{<i}} \mathrm{D}_{S_{<i}}$, as verified in the proof of Lemma 3.16. In either case the result is non-zero. A similar argument now shows that this term can not be canceled by other summands of $\mathrm{D}_{i} z \mathrm{e}_{v-1}$. Thus, we have $z \mathrm{D}_{i} \mathrm{e}_{v-1} \neq 0$, in contradiction to the minimality of $v$.

Now define $z^{\prime}=z-c \mathrm{~L}_{v}$. By Lemma 3.17 we have $z^{\prime} \in Z\left(\overline{\mathrm{Z}}_{e-1}\right)$ and by Corollary 3.11 we know that $z^{\prime}$ is supported at vertices $w>v$. Now proceed by induction.

\section{The Center of the tilting Category}

We now explain how $Z(\overline{\mathbf{Z}})$ and $\mathbf{Z}($ Tilt $)$ are related.

4A. Some general (and well-known) facts. Let $\mathbb{k}$ denote any commutative unital ring. For an additive $\mathbb{k}$-linear category $\mathbf{C}$ we let $\operatorname{End}(\mathbf{C})$ denote its category of $\mathbb{k}$-linear endofunctors (which are thus automatically additive) and natural transformations. Within this category we

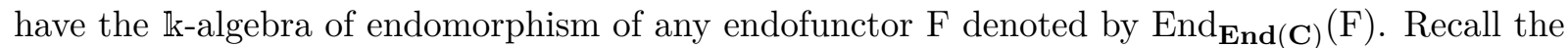
following definition.

Definition 4.1 Let $\mathbf{C}$ be an additive $\mathbb{k}$-linear category. Then its center $\mathbf{Z}(\mathbf{C})$ is defined as

$$
\mathbf{Z}(\mathbf{C})=\operatorname{End}_{\mathbf{E n d}(\mathbf{C})}\left(\operatorname{Id}_{\mathbf{C}}\right),
$$

i.e. the natural transformations of the identity functor $\operatorname{Id}_{\mathbf{C}}$ of $\mathbf{C}$.

Lemma 4.2 We have the following.

(a) The space $\mathbf{Z}(\mathbf{C})$ is a commutative $\mathbb{k}$-algebra.

(b) If $\mathbf{C}$ and $\mathbf{D}$ are equivalent additive $\mathbb{k}$-linear categories, then $\mathbf{Z}(\mathbf{C}) \cong \mathbf{Z}(\mathbf{D})$ as commutative $\mathbb{R}$-algebras.

(c) If $\mathbf{C} \cong \prod_{i \in I} \mathbf{C}_{i}$ as additive $\mathbb{k}$-linear categories with trivial hom spaces between $\mathbf{C}_{i}$ and $\mathbf{C}_{j}$ unless $i=j$, then $\mathbf{Z}(\mathbf{C}) \cong \prod_{i \in I} \mathbf{Z}\left(\mathbf{C}_{i}\right)$ as commutative $\mathbb{k}$-algebras.

Proof. The first claim is evident. For the second claim we observe that, for any additive $\mathbb{k}$-linear full subcategory $\mathbf{D} \subset \mathbf{C}$, restriction defines a $\mathbb{k}$-algebra homomorphism $r: \mathbf{Z}(\mathbf{C}) \rightarrow \mathbf{Z}(\mathbf{D})$. If $\mathbf{C}$ and $\mathbf{D}$ are equivalent then one can check that $r$ is an isomorphism. The final claim can be proven mutatis mutandis as the second claim.

Let us consider the example $p$ Mod-A (projective right modules of some $\mathbb{k}$-algebra A).

Lemma 4.3 Let A be a $\mathbb{k}$-algebra.

(a) We have an inclusion $Z(\mathrm{~A}) \hookrightarrow \mathbf{Z}(p \mathbf{M o d}-\mathrm{A})$ of commutative $\mathbb{k}$-algebras. 
(b) If $\mathrm{A}$ is unital, then we have an isomorphism $Z(\mathrm{~A}) \stackrel{\cong}{\rightrightarrows} \mathbf{Z}(p \mathbf{M o d}-\mathrm{A})$ of commutative $\mathbb{k}$-algebras.

Proof. We claim that the map

$$
Z(\mathrm{~A}) \ni c \mapsto \chi_{c}:=\left\{\chi_{\mathrm{P}}: \mathrm{P} \rightarrow \mathrm{P}, m \mapsto m c \mid \mathrm{P} \in p \text { Mod-A }\right\}
$$

gives the required inclusion or isomorphism, respectively.

Indeed, it is easy to see that $\chi_{c}$ is actually in $\mathbf{Z}(p$ Mod-A $)$ and that this map is a well-defined inclusion of $\mathbb{k}$-algebras.

To prove surjectivity under the assumption of unitality of $\mathrm{A}$, given $\chi \in \mathbf{Z}(p \mathbf{M o d}-\mathrm{A})$, its value on the projective right $\mathrm{A}$-module $\mathrm{A}$ is by definition a map of right $\mathrm{A}$-modules $\chi_{\mathrm{A}}: \mathrm{A} \rightarrow \mathrm{A}$. Let $c:=\chi_{\mathrm{A}}(1) \in \mathrm{A}$, which actually belongs to $Z(\mathrm{~A})$. Next, for any $\mathrm{P} \in p \mathbf{M o d}-\mathrm{A}$ and any $m \in \mathrm{P}$ there is a unique $f \in \operatorname{Hom}_{p \operatorname{Mod}-\mathrm{A}}(\mathrm{A}, \mathrm{P})$ with $f(1)=m$. Using this and naturality, we get

$$
\chi_{\mathrm{P}}(m)=\chi_{\mathrm{P}}(f(1))=f\left(\chi_{\mathrm{A}}(1)\right)=f(c)=m c,
$$

which proves surjectivity.

4B. The tilting center. Let us modify Lemma 4.3, which we need to do since $\mathrm{Z}$ is non-unital:

Lemma 4.4 We have an isomorphism of commutative $\mathbb{K}$-algebras given by

$$
Z(\overline{\mathbf{Z}}) \stackrel{\cong}{\rightarrow} \mathbf{Z}(p \text { Mod-Z }), \quad Z(\overline{\mathrm{Z}}) \ni c \mapsto \chi_{c}:=\left\{\chi_{\mathrm{P}}: \mathrm{P} \rightarrow \mathrm{P}, m \mapsto m c \mid \mathrm{P} \in p \text { Mod-Z }\right\} .
$$

Proof. Defining $c:=\chi_{\bar{z}}\left(\sum_{v \in \mathbb{N}} \mathbf{e}_{v-1}\right)$, the argument is the same as in Lemma 4.3.

By the well-understood block decomposition

$$
\text { Tilt }=\bigoplus_{e \in E v e} \text { Tilt }_{e-1}, \quad \text { Tilt }_{e-1}=\left\{\mathrm{T}(v-1) \mid v \in(e)_{\mathrm{p}}\right\},
$$

where hom spaces between Tilt $_{e-1}$ and Tilt $_{e^{\prime}-1}$ are trivial unless $e=e^{\prime}$.

Definition 4.5 For $v \in(e)_{\mathrm{p}} \backslash\{e\}$ we define the natural transformation

$$
\chi_{v}:=\left\{\chi_{\mathrm{T}}: \mathrm{T} \rightarrow \mathrm{T}, \chi_{\mathrm{T}}=\mathrm{L}_{v} \mid \mathrm{T} \in \mathbf{T i l t}_{e-1}\right\},
$$

where $\mathrm{L}_{v}$ was defined in Definition 3.12 .

Thus, we can finally prove Theorem A, which is a consequence of:

Theorem 4.6 For $e \in \mathrm{Eve}^{<\mathrm{p}}$ we have an $\mathbb{K}$-algebra isomorphism

$$
\mathbf{Z}\left(\text { Tilt }_{e-1}\right) \stackrel{\cong}{\rightrightarrows} \mathbb{K}\left[X_{v} \mid v \in(e)_{\mathbf{p}} \backslash\{e\}\right] /\left\langle X_{v} X_{w} \mid v, w \in(e)_{\mathbf{p}} \backslash\{e\}\right\rangle, \quad \chi_{v} \mapsto X_{v} .
$$

Proof. By Theorem 2.14 and Lemma 4.2.(b) we need to compute $\mathbf{Z}\left(p \mathbf{M o d}-\mathbf{Z}_{e-1}\right)$. This in turn, by Lemma 4.4, reduces to compute the algebra center of $\bar{Z}_{e-1}$, which is Theorem 3.13.

4C. The center and Donkin's tensor product theorem. We expect that the central elements $\mathrm{L}_{v}$ admit an interpretation via Donkin's tensor product theorem, which, in our notation, takes the following form.

Proposition 4.7 (See [Don93, Proposition 2.1].) For $v=\left[a_{j}, \ldots, a_{0}\right]_{\mathrm{p}}$ we have an isomorphism of (left) $\mathrm{SL}_{2}(\mathbb{K})$-modules

$$
\mathrm{T}(v-1) \cong \mathrm{T}\left(a_{j}-1\right)^{j} \otimes\left(\bigotimes_{i=0}^{j-1} \mathrm{~T}\left(a_{i}+\mathrm{p}-1\right)^{i}\right),
$$

where the superscripts indicate Frobenius twist. 
Lemma 4.8 Let $v=\left[a_{j}, \ldots, a_{0}\right]_{\mathrm{p}}$ and $v^{\prime}=\left[1, a_{j-1}, \ldots, a_{0}\right]_{\mathrm{p}}$. For all $w=\left[b_{k}, \ldots, b_{j}, b_{j-1}, \ldots, b_{0}\right]_{\mathrm{p}} \in$ $(v)_{\mathrm{p}}^{\odot}$, define $w^{\prime}=\left[b_{k}, \ldots, b_{j}\right]_{\mathrm{p}}$. We have an isomorphism of (left) $\mathrm{SL}_{2}(\mathbb{K})$-modules

$$
\mathrm{T}(w-1) \cong \mathrm{T}\left(w^{\prime}-1\right)^{j} \otimes \mathrm{T}\left(v^{\prime}-1\right) .
$$

Proof. First, note that Lemma 3.10 gives $w=\left[b_{k}, \ldots, b_{j+1}, b_{j}, a_{j-1}, \ldots, a_{0}\right]_{\mathrm{p}}$. Thus, applying Proposition 4.7 proves the claim.

Example 4.9 As before, let $\mathrm{p}=7, v=[3,1,6,5,0,5,6]_{7}$ with $v^{\prime}=[1,1,6,5,0,5,6]_{7}$. Then $w=[1,4,1,6,5,0,5,6]_{7} \in(v)_{\mathrm{p}}^{\odot}$ and $w^{\prime}=[1,4]_{7}$. Proposition 4.7 and Lemma 4.8 give

$$
\begin{gathered}
\mathrm{T}\left(v^{\prime}-1\right) \cong \mathrm{T}(7)^{5} \otimes \mathrm{T}(12)^{4} \otimes \mathrm{T}(11)^{3} \otimes \mathrm{T}(6)^{2} \otimes \mathrm{T}(11)^{1} \otimes \mathrm{T}(12)^{0}, \\
\mathrm{~T}(v-1) \cong \mathrm{T}(2)^{6} \otimes \mathrm{T}\left(v^{\prime}-1\right), \\
\mathrm{T}(w-1) \cong \mathrm{T}\left(w^{\prime}-1\right)^{6} \otimes \mathrm{T}\left(v^{\prime}-1\right) \cong \mathrm{T}(0)^{7} \otimes \mathrm{T}(10)^{6} \otimes \mathrm{T}\left(v^{\prime}-1\right) .
\end{gathered}
$$

In the situation of Lemma 4.8 where

$$
\mathrm{T}(w-1) \cong \mathrm{T}\left(w^{\prime}-1\right)^{j} \otimes \mathrm{T}\left(v^{\prime}-1\right)
$$

it seems plausible that $\mathrm{L}_{v} \mathrm{e}_{w-1}=c \cdot \mathrm{id}_{\mathrm{T}\left(w^{\prime}-1\right)^{j}} \otimes \mathrm{L}_{v^{\prime}}$ for some scalar $c \in \mathbb{K}$. One approach to proving this statement involves giving a diagrammatic interpretation of Frobenius twists in the Temperley-Lieb category. Alternatively, it would also be interesting to have a proof that such elements are indeed central and span the center, which does not use the explicit presentation of Tilt, e.g. using the abstract basis given in [AST18].

4D. Blocks via the center. Let us discuss a classical application of the center of a category, mimicking the well-understood case of category $\mathcal{O}$. Note that $\mathbf{Z}(\mathbf{C})$ acts naturally on all objects $\mathbf{X}$ of $\mathbf{C}$ via

$$
\chi \cdot \mathrm{f}:=\mathrm{f} \circ \chi_{\mathrm{x}}=\chi_{\mathrm{x}} \circ \mathrm{f}, \quad \chi \in \mathbf{Z}(\mathbf{C}), \mathrm{f} \in \operatorname{End}_{\mathbf{C}}(\mathrm{X}) .
$$

In particular, for any character of the center $\chi_{s}: \mathbf{Z}(\mathbf{C}) \rightarrow \mathbb{K}$, we can fiber the category $\mathbf{C}$ by defining full subcategories $\mathbf{C}_{s} \subset \mathbf{C}$ consisting of all objects where $\mathbf{Z}(\mathbf{C})$ acts with character $\chi_{s}$.

By Theorem A, the $\mathbf{C}_{s}$ for $\mathbf{C}=$ Tilt are exactly the blocks $\mathbf{T i l t}{ }_{e-1}$, which follows because each $\mathbb{K}$-algebra $\mathbf{Z}\left(\right.$ Tilt $\left._{e-1}\right)$, which has only one non-nilpotent basis element, has exactly one simple.

4E. The Casimir element. Let $C: A \rightarrow \mathbb{K}$ denote the Casimir element of $\mathrm{SL}_{2}(\mathbb{K})$, which is an element in its distribution algebra, i.e. a map from the coordinate ring $A$ of $\mathrm{SL}_{2}(\mathbb{K})$ to $\mathbb{K}$. Recall that $C$ acts on every $\mathrm{SL}_{2}(\mathbb{K})$ module $\mathrm{M}$ using the coaction $\Delta_{\mathrm{M}}$ of $A$ on $\mathrm{M}$ :

$$
C: \mathrm{M} \stackrel{\Delta_{\mathrm{M}}}{\longrightarrow} \mathrm{M} \otimes_{\mathbb{K}} A \stackrel{1 \otimes C}{\longrightarrow} \mathrm{M} \otimes_{\mathbb{K}} \mathbb{K} \stackrel{\cong}{\rightrightarrows} \mathrm{M} .
$$

Let $\mathrm{L}(v-1)$ denote the simple $\mathrm{SL}_{2}(\mathbb{K})$ module of highest weight $v-1$. We choose $C=(h+1)^{2}+4 f e$ and see that $C$ acts on $\mathrm{L}(v-1)$ as $v^{2} \mathrm{id}$. The proof of Lemma 3.14 implies that $C$ acts on $\mathrm{L}(v-1)$ as multiplication by $v^{2}=a_{0}^{2}=\left(\mathrm{p}-a_{0}\right)^{2}=e^{2} \in \mathbb{F}_{\mathrm{p}}$ for every $v \in(e)_{\mathrm{p}}$. Since all $L(v-1)$ that appear in composition series of objects of Tilt $_{e-1}$ have $v \in(e)_{\mathrm{p}}$, we see that $C$ acts by this scaling on all objects in Tilt $_{e-1}$. Hence, in the blocks $Z\left(\overline{\mathbf{Z}}_{e-1}\right)$ of the Ringel dual we have $C=e^{2} \cdot 1$.

\section{TWO OTHER CASES}

Remark 5.1 The two theorems below can be proven, mutatis mutandis, as for Tilt, but the computations are much simpler. Thus, we decided to keep the proofs short. 
5A. A variation: the quantum case (generation 1 ). In the quantum group case the same calculations, using the Ringel dual computed in [AT17], work, but are much simpler, and so is the result. Let Tilt $^{q}$ denote the quantum analog of Tilt with quantum parameter $q$, where we use Lusztig's divided power quantum group. Throughout this part, [AT17] serves as our reference for statements about Tilt ${ }^{q}$. However, we use the notation from this paper. In particular, we write $\left[a_{j}, \ldots, a_{0}\right]_{k}:=\sum_{i=0}^{j} a_{i} k^{i}=v$ for the $k$-adic expansion with digits $0 \leq a_{i}<k$.

We will work over a field $\mathbb{C}(q)$ and distinguish the following cases:

- The quantum parameter $q$ is a formal parameter, or $q \in \mathbb{C}$ is $q= \pm 1$ or not a root of unity. Here we set $k=\infty$.

- The quantum parameter $q \in \mathbb{C}$ is a root of unity $q \neq \pm 1$. Here $k$ denotes the order of $q^{2}$.

We also let

$$
\chi_{v}:=\left\{\chi_{\mathrm{T}}: \mathrm{T} \rightarrow \mathrm{T}, \chi_{\mathrm{T}}=\mathrm{L}_{v}^{\{0\}} \mid \mathrm{T} \in \text { Tilt }\right\}, \quad \delta_{v}:=\left\{\delta_{\mathrm{T}}: \mathrm{T} \rightarrow \mathrm{T}, \delta_{\mathrm{T}}=\delta_{v} \mid \mathrm{T} \in \text { Tilt }\right\},
$$

where $\mathrm{L}_{v}^{\{0\}}$ and $\delta_{v}$ are the maps that act as non-zero only on summands $\mathrm{T}(v-1)$, on which they are a head-to-socle map and the identity, respectively.

Theorem 5.2 We have the following.

(a) For $k=\infty$ there is an isomorphism of $\mathbb{C}(q)$-algebras

$$
\mathbf{Z}\left(\text { Tilt }^{q}\right) \stackrel{\cong}{\rightarrow} \prod_{\mathbb{N}} \mathbb{C}(q), \quad \delta_{v} \mapsto 1_{v} .
$$

(b) For the root of unity case there is an isomorphism of $\mathbb{C}$-algebras

$$
\mathbf{Z}\left(\mathbf{T i l t}^{q}\right) \stackrel{\cong}{\rightarrow} \bigoplus_{i=-1}^{k-2} \mathrm{X}(i), \quad \mathrm{X}(i)= \begin{cases}\prod_{\mathbb{N}} \mathbb{C} & \text { if } i=-1, \\ \mathbb{C}\left[X_{v} \mid v \in \mathbb{N}\right] /\left\langle X_{v} X_{w} \mid v, w \in \mathbb{N}\right\rangle & \text { otherwise, }\end{cases}
$$

where $\chi_{w} \mapsto X_{v}$, and $\delta_{x} \mapsto 1_{v}$ for $w, x \in \mathbb{N}$ as explained in the proof.

Proof. (a). This claim is clear as Tilt ${ }^{q}$ is semisimple and its simples are indexed by $\mathbb{N}$.

(b). For the root of unity case we recall that we have equivalences of additive $\mathbb{K}$-linear categories

$$
\begin{gathered}
\text { Tilt }^{q} \cong \text { Tilt }_{S t}^{q} \oplus \text { Tilt }_{0}^{q} \oplus \ldots \oplus \text { Tilt }_{k-2}^{q}, \\
\text { Tilt }_{0}^{q} \cong \ldots \cong \text { Tilt }_{k-2}^{q} \cong p \text { Mod-Z }
\end{gathered}
$$

where Tilt $_{S t}^{q}$ is semisimple with simple objects being the indecomposable quantum tilting modules whose highest weight $v$ satisfies $a_{0}=0$, which is the first case in (5-2).

Moreover, the Ringel dual $\mathrm{Z}^{q}$ of Tilt $_{0}^{q}$ is a zigzag algebra with a boundary condition on the vertex set $\mathbb{N}$, i.e.

$$
\left(v_{0}-1\right) \underset{\mathrm{D}_{\{0\}}}{\stackrel{\mathrm{U}_{\{0\}}}{\rightleftarrows}}\left(v_{1}-1\right) \underset{\mathrm{D}_{\{0\}}}{\stackrel{\mathrm{U}_{\{0\}}}{\rightleftarrows}}\left(v_{2}-1\right) \underset{\mathrm{D}_{\{0\}}}{\stackrel{\mathrm{U}_{\{0\}}}{\rightleftarrows}}\left(v_{3}-1\right) \underset{\mathrm{D}_{\{0\}}}{\stackrel{\mathrm{U}_{\{0\}}}{\rightleftarrows}} \ldots,
$$

where $v_{0}=1$ and $v_{i+1}=v_{i}(0)$ for $i \geq 0$, subject to the relations

$\mathrm{D}_{\{0\}} \mathrm{D}_{\{0\}} \mathrm{e}_{v-1}=0, \mathrm{U}_{\{0\}} \mathrm{U}_{\{0\}} \mathrm{e}_{v-1}=0, \mathrm{D}_{\{0\}} \mathrm{U}_{\{0\}} \mathbf{e}_{v-1}=\mathrm{U}_{\{0\}} \mathrm{D}_{\{0\}} \mathrm{e}_{v-1}$ for $v \neq 1, \mathrm{D}_{\{0\}} \mathrm{U}_{\{0\}} \mathrm{e}_{0}=0$.

(Note the boundary relation $\mathrm{D}_{\{0\}} \mathrm{U}_{\{0\}} \mathrm{e}_{0}=0$.) Formally, the algebra $\mathrm{Z}^{q}$ is generated by $\mathrm{e}_{v-1}$ for $v \in \mathbb{N}$ being of the form $v=1(0) \ldots(0)$, and elements $\mathrm{D}_{\{0\}} \mathbf{e}_{v-1}$ and $\mathrm{U}_{\{0\}} \mathrm{e}_{v-1}$, modulo the relations shown above, which are the analogs of the relations in Theorem 2.14.

Observe that we have central elements in $\mathrm{Z}^{q}$ of the form

$$
\mathrm{L}_{v}^{\{0\}}=\mathrm{D}_{\{0\}} \mathrm{U}_{\{0\}} \mathbf{e}_{v-1}=\mathrm{U}_{\{0\}} \mathrm{D}_{\{0\}} \mathbf{e}_{v-1} \text {, for } v \neq 1 \text {. }
$$


These are central since they are annihilated by any element except their idempotent. Moreover, no other element is central, implying the second case in (5-2), using arguments as in Section 4A and the quantum analog of Lemma 4.4. In particular, $\mathrm{L}_{v}^{\{0\}}$ corresponds to the natural transformation in (5-1) and satisfies the relations of $X_{v}$ in $\mathbb{C}\left[X_{v} \mid v \in \mathbb{N}\right] /\left\langle X_{v} X_{w} \mid v, w \in \mathbb{N}\right\rangle$.

5B. A variation: the $G_{g} T$ case for $g=1,2$ (generations 1,2 ). Recall that $\left[a_{j}, \ldots, a_{0}\right]_{\mathrm{p}}=$ $\sum_{i=0}^{j} a_{i} \mathrm{p}^{i}=v$. Using the same methods, in the case of projective $G_{g} T$-modules for $\mathrm{SL}_{2}(\mathbb{K})$ and $g=1,2$ one can also calculate the center of the corresponding additive $\mathbb{K}$-linear category $G_{g} T-p$ Mod. The corresponding Ringel duals were computed in [An19], which is also our reference for statements about $G_{g} T-p$ Mod. Again, the resulting algebras are much simpler than for Tilt.

Before we can state the theorem, let us define natural transformations $\mathrm{L}_{v}^{\{0\}}$ and $\delta_{v}$ mutatis mutandis as in (5-1). We also need

$$
\begin{gathered}
\chi_{v}^{X}:=\left\{\chi_{\mathrm{P}}^{X}: \mathrm{P} \rightarrow \mathrm{P}, \chi_{\mathrm{P}}^{X}=\mathrm{L}_{v}^{\{0\}} \mathrm{L}_{v}^{\{1\}} \mid \mathrm{P} \in G_{2} T-p \text { Mod }\right\}, \\
\chi_{x}^{Y}:=\left\{\chi_{\mathrm{P}}^{Y}: \mathrm{P} \rightarrow \mathrm{P}, \chi_{\mathrm{P}}^{Y}=\mathrm{L}_{x}^{\{0\}} \mid \mathrm{P} \in G_{2} T-p \text { Mod }\right\},
\end{gathered}
$$

where both, $\mathrm{L}_{v}^{\{0\}} \mathrm{L}_{v}^{\{1\}}$ and $\mathrm{L}_{x}^{\{0\}}$, are non-zero only on corresponding indecomposable projective $G_{2} T$-modules $\mathrm{P}(v-1)$ and $\mathrm{P}(x-1)$ for $v \in \mathbb{Z} \backslash \mathrm{p} \mathbb{Z}$ and $x \in \mathrm{p} \mathbb{Z}$, where they are the head-to-socle maps. We further need an analog of (4-1), namely

$$
\chi_{i}^{Z}:=\left\{\chi_{\mathrm{P}}^{Z}: \mathrm{P} \rightarrow \mathrm{P}, \chi_{\mathrm{P}}^{Z}=\mathrm{L}_{i} \mid \mathrm{P} \in G_{2} T-p \mathbf{M o d}\right\},
$$

where $\mathrm{L}_{i}$ is only zero on all indecomposable summands $\mathrm{P}(j-1)$ for $j \in r(i)$ (the row of $i$, cf. (5-5)). On such $\mathrm{P}(j-1)$ it is a map that factors through the highest weights of its horizontal neighbors (on the grid as in (5-5)), which is unique up to scalars.

Theorem 5.3 We have the following.

(a) For $g=1$ we have $\mathbb{K}$-algebra isomorphisms

$$
\mathbf{Z}\left(G_{1} T \text {-p } \mathbf{M o d}\right) \stackrel{\cong}{\rightarrow} \bigoplus_{i=-1}^{k-2} \mathrm{X}(i), \mathrm{X}(i)= \begin{cases}\prod_{\mathbb{Z}} \mathbb{K} & \text { if } i=-1 \\ \mathbb{K}\left[X_{v} \mid v \in \mathbb{Z}\right] /\left\langle X_{v} X_{w} \mid v, w \in \mathbb{Z}\right\rangle & \text { otherwise }\end{cases}
$$

where $\chi_{w} \mapsto X_{v}$, and $\delta_{x} \mapsto 1_{v}$ for $w, x \in \mathbb{Z}$ as explained in the proof.

(b) For $g=2$ we have $\mathbb{K}$-algebra isomorphisms

$$
\mathbf{\mathrm { X }}(i) \cong \begin{cases}\mathbf{Z}\left(G_{1} T \text {-pMod }\right) & \text { if } i=-1, \\ \mathbb{K}\left[X_{v}, Y_{x}, Z_{i} \mid v, x \in \mathbb{Z}, i \in \mathrm{Eve}^{<\mathrm{p}}\right] / \mathrm{I} & \text { otherwise },\end{cases}
$$

where

$$
\mathrm{I}=\left\langle\begin{array}{c|c}
X_{v} X_{w}, X_{v} Y_{y}, X_{v} Z_{j}, & v, w \in \mathbb{Z} \\
Y_{x} X_{w}, Y_{x} Y_{y}, Y_{x} Z_{j}, & x, y \in \mathbb{Z}, \\
Z_{i} X_{w}, Z_{i} Y_{y}, Z_{i} Z_{j}, & i, j \in \mathrm{Eve}^{<\mathrm{p}}
\end{array}\right\rangle
$$

and $\mathrm{L}_{w}^{X} \mapsto X_{v}$, and $\mathrm{L}_{y}^{Y} \mapsto Y_{x}$ for $w, y \in \mathbb{Z}$, and $\mathrm{L}_{j}^{Z} \mapsto Z_{i}$ for $j \in \mathrm{Eve}^{<\mathrm{p}}$ as explained in the proof.

Proof. (a). Very similar as in the quantum case we have a decomposition of additive $\mathbb{K}$-linear categories

$$
\begin{gathered}
G_{1} T-p \mathbf{M o d} \cong G_{1} T-p \mathbf{M o d}_{S t} \oplus G_{1} T-p \mathbf{M o d}_{0} \oplus \ldots \oplus G_{1} T-p \mathbf{M o d}_{\mathrm{p}-2} \\
G_{1} T-p \mathbf{M o d}_{0} \cong \ldots \cong G_{1} T-p \mathbf{M o d}_{\mathrm{p}-2} \cong p \operatorname{Mod}-\mathrm{Z}^{G_{1} T}
\end{gathered}
$$


where $G_{2} T$ - $p \mathbf{M o d}_{S t}$ is semisimple with simple objects being projective $G_{1} T$-modules indexed as in the first case in (5-3). The Ringel dual quiver algebra $Z^{G_{1} T}$ of $G_{1} T-p \mathbf{M o d}_{0}$ in this case is a zigzag algebra on the vertex set $\mathbb{Z}$, i.e.

$$
\ldots \frac{\mathrm{U}_{\{0\}}}{\underset{\mathrm{D}_{\{0\}}}{\rightleftarrows}}\left(v_{-1}-1\right) \underset{\mathrm{D}_{\{0\}}}{\stackrel{\mathrm{U}_{\{0\}}}{\rightleftarrows}}\left(v_{0}-1\right) \stackrel{\mathrm{U}_{\{0\}}}{\underset{\mathrm{D}_{\{0\}}}{\rightleftarrows}}\left(v_{1}-1\right) \underset{\mathrm{D}_{\{0\}}}{\stackrel{\mathrm{U}_{\{0\}}}{\rightleftarrows}}\left(v_{2}-1\right) \stackrel{\mathrm{U}_{\{0\}}}{\underset{\mathrm{D}_{\{0\}}}{\rightleftarrows}} \ldots,
$$

where $v_{0}=1$ and $v_{i+1}=v_{i}(0)$ for $i \in \mathbb{N}_{0}, v_{-i}=-v_{i}+2$ for $i$ even, and $v_{-i}=-v_{i}+2 \mathrm{p}-2$ for $i$ odd. These are the numbers with $\mathrm{p}$-adic expansion $\left[n_{1}, a_{0}\right]_{\mathrm{p}}$ where $a_{0} \in\{0, \ldots, \mathrm{p}-1\}$ and $n_{1} \in \mathbb{Z}$, which can be reached from 1 by successive upward or downward reflection in the zeroth digit. The generators $\mathrm{D}_{\{0\}}$ and $\mathrm{U}_{\{0\}}$ are subject to the relations

$$
\mathrm{D}_{\{0\}} \mathrm{D}_{\{0\}} \mathrm{e}_{v-1}=0, \mathrm{U}_{\{0\}} \mathrm{U}_{\{0\}} \mathbf{e}_{v-1}=0, \mathrm{D}_{\{0\}} \mathrm{U}_{\{0\}} \mathbf{e}_{v-1}=\mathrm{U}_{\{0\}} \mathrm{D}_{\{0\}} \mathrm{e}_{v-1} \text {. }
$$

Formally, the algebra $\mathrm{Z}^{G_{1} T}$ is generated by $\mathrm{e}_{v-1}$ for $v \in \mathbb{Z}$ being of the form $v= \pm(1(0) \ldots(0))$, and elements $\mathrm{D}_{\{0\}} \mathbf{e}_{v-1}$ and $\mathrm{U}_{\{0\}} \mathbf{e}_{v-1}$. The relations are the ones above together with the usual idempotent relations. Thus, the situation is analogous to the quantum case. In particular, the same arguments, mutatis mutandis, as for the quantum group give the second case in (5-3).

(c). We start by recalling that

$$
\begin{gathered}
G_{2} T-p \mathbf{M o d} \cong G_{2} T-p \mathbf{M o d}_{S t} \oplus G_{2} T-p \mathbf{M o d}_{0} \oplus \ldots \oplus G_{2} T-p \mathbf{M o d}_{\mathrm{p}-2}, \\
G_{2} T-p \mathbf{M o d}_{S t} \cong G_{1} T-p \mathbf{M o d}, \quad G_{2} T-p \mathbf{M o d}_{0} \cong \ldots \cong G_{2} T-p \operatorname{Mod}_{\mathfrak{p}-2} \cong p \operatorname{Mod}^{G_{2} T},
\end{gathered}
$$

where $G_{2} T-p \mathbf{M o d}_{S t}$ is no longer semisimple, but rather equivalent to $G_{1} T-p$ Mod. This gives us the first summand in (5-4). The Ringel dual quiver algebra $Z^{G_{2} T}$ still has vertex set $\mathbb{Z}$, but arranged on a grid, e.g. if $\mathrm{p}=5$, then, as explained in [An19, Section 6.3]:

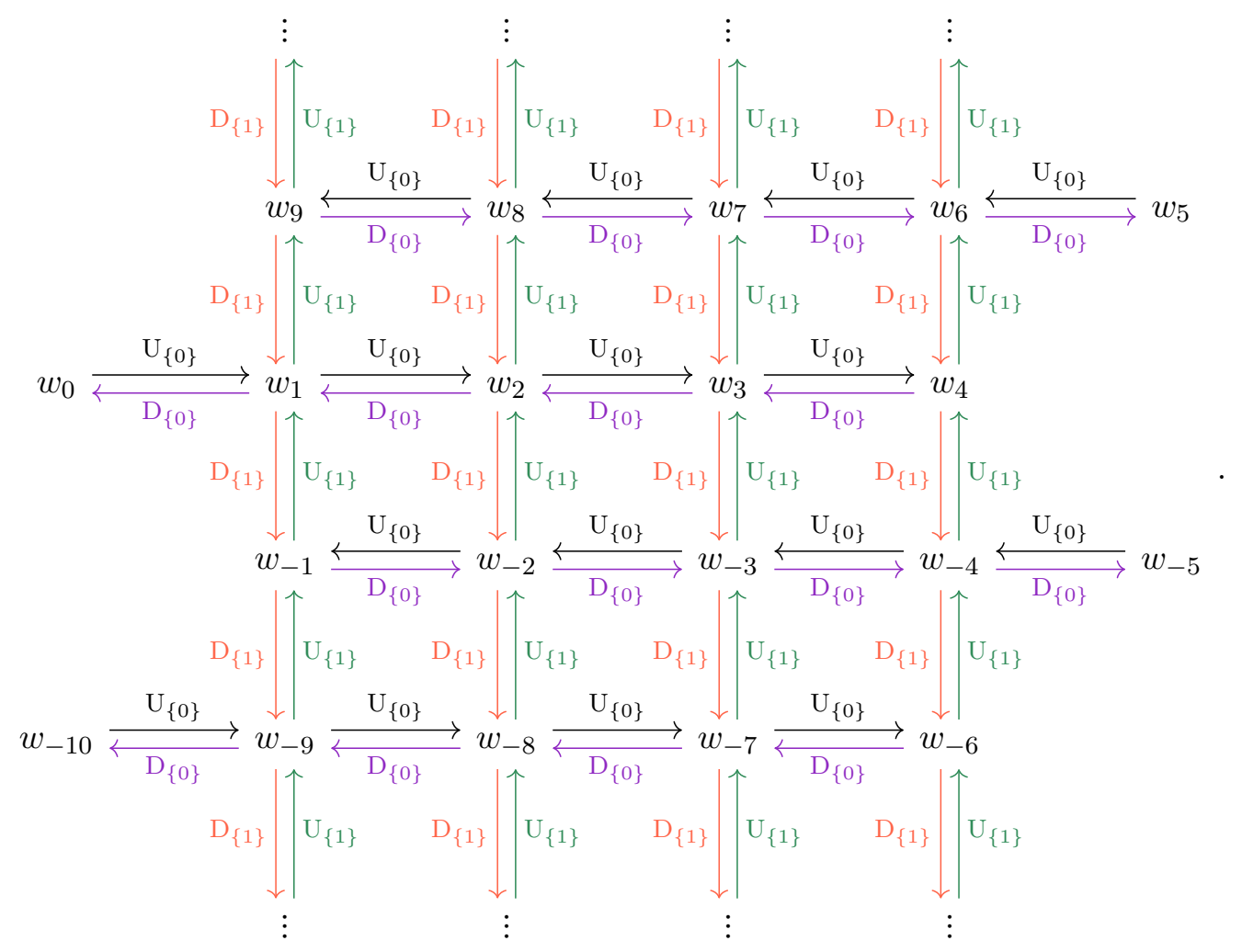

The indexing of the positively labeled vertices $w_{i}=v_{i}-1$ hereby works as follows. $v_{0}=[1]_{5}(=1)$, and each horizontal step is (0), e.g. $v_{1}=[1]_{5}(0)=[2,-1]_{5}=[1,4]_{5}(=9)$, while each vertical step is (1), e.g. $v_{9}=[1,4]_{5}(1)=[2,-1,4]_{5}(=49)$. Moreover, $w_{-i}=-w_{i}$, if $i \geq 0$ is even, $w_{-i}=-w_{i}+2 \mathrm{p}-4$, if $i>0$ is odd. The $v_{i}$ are exactly the integers with p-adic expansion 
$\left[n_{2}, a_{1}, a_{0}\right]_{\mathrm{p}}$ where $a_{0}, a_{1} \in\{0, \ldots, \mathrm{p}-1\}$ and $n_{2} \in \mathbb{Z}$, which can be reached from 1 by reflection in the first two digits.

Precisely, the algebra $\mathrm{Z}^{G_{2} T}$ is generated by $\mathrm{e}_{v-1}$ for $v \in \mathbb{Z}$ being as above, and elements $\mathrm{D}_{\{0\}} \mathrm{e}_{v-1}$ and $\mathrm{U}_{\{0\}} \mathbf{e}_{v-1}$ as well as $\mathrm{D}_{\{1\}} \mathbf{e}_{v-1}$ and $\mathrm{U}_{\{1\}} \mathbf{e}_{v-1}$. The relations are such that each column and each row is a zigzag algebra, and all squares commute, i.e.:

(1) Each row is a zigzag algebra, cf. Theorem 2.14.(2) and (6), i.e.

$$
\mathrm{U}_{\{0\}} \mathrm{U}_{\{0\}} \mathrm{e}_{v-1}=0=\mathrm{D}_{\{0\}} \mathrm{D}_{\{0\}} \mathrm{e}_{v-1}, \quad \mathrm{D}_{\{0\}} \mathrm{U}_{\{0\}} \mathrm{e}_{v-1}=\mathrm{U}_{\{0\}} \mathrm{D}_{\{0\}} \mathrm{e}_{v-1} .
$$

(No boundary condition.)

(2) Each column is a zigzag algebra, $c f$. Theorem 2.14.(2) and (6), i.e.

$$
\mathrm{U}_{\{1\}} \mathrm{U}_{\{1\}} \mathrm{e}_{v-1}=0=\mathrm{D}_{\{1\}} \mathrm{D}_{\{1\}} \mathrm{e}_{v-1}, \quad \mathrm{D}_{\{1\}} \mathrm{U}_{\{1\}} \mathrm{e}_{v-1}=\mathrm{U}_{\{1\}} \mathrm{D}_{\{1\}} \mathrm{e}_{v-1} .
$$

(3) All squares commute, cf. Theorem 2.14.(4), i.e.

$$
\begin{aligned}
& \mathrm{D}_{\{1\}} \mathrm{U}_{\{0\}} \mathbf{e}_{v-1}=\mathrm{D}_{\{0\}} \mathrm{D}_{\{1\}} \mathbf{e}_{v-1}, \quad \mathrm{D}_{\{0\}} \mathrm{U}_{\{1\}} \mathbf{e}_{v-1}=\mathrm{U}_{\{1\}} \mathrm{U}_{\{0\}} \mathbf{e}_{v-1}, \\
& \mathrm{D}_{\{1\}} \mathrm{D}_{\{0\}} \mathbf{e}_{v-1}=\mathrm{D}_{\{0\}} \mathrm{U}_{\{1\}} \mathbf{e}_{v-1}, \quad \mathrm{D}_{\{0\}} \mathrm{D}_{\{1\}} \mathbf{e}_{v-1}=\mathrm{D}_{\{1\}} \mathrm{U}_{\{0\}} \mathbf{e}_{v-1} .
\end{aligned}
$$

These hold only for complete squares, i.e.

$$
\left.w_{0} \stackrel{\mathrm{U}_{\{0\}}}{\longrightarrow}\right|_{w_{1}} ^{w_{\{1\}},}
$$

as in (5-5), does not satisfy any relation, and is in particular, not zero.

Hence, we get row and column loops

$$
\begin{aligned}
\mathrm{L}_{i} & :=\mathrm{L}_{\{0\}} \mathrm{e}_{v_{i}-1}=\mathrm{D}_{\{0\}} \mathrm{U}_{\{0\}} \mathrm{e}_{v_{i}-1}=\mathrm{U}_{\{0\}} \mathrm{D}_{\{0\}} \mathrm{e}_{v_{i}-1}, \\
\mathrm{~L}_{i}^{\prime} & :=\mathrm{L}_{\{1\}} \mathbf{e}_{v_{i}-1}=\mathrm{D}_{\{1\}} \mathrm{U}_{\{1\}} \mathbf{e}_{v_{i}-1}=\mathrm{U}_{\{1\}} \mathrm{D}_{\{1\}} \mathbf{e}_{v_{i}-1} .
\end{aligned}
$$

(Here, as in (5-5), only one expression for $\mathrm{L}_{\{0\}} \mathrm{e}_{v_{i}-1}$ makes sense if $\mathrm{p} \mid i$. The above is just a shorthand notation.) The relations imply that

$$
\mathrm{e}_{v_{i}-1} \mathrm{Z}^{G_{2} T} \mathrm{e}_{v_{i}-1} \cong \begin{cases}\mathbb{K}\left[\mathrm{L}_{i}\right] /\left\langle\mathrm{L}_{i}^{2}\right\rangle & \text { if } \mathrm{p} \mid i, \\ \mathbb{K}\left[\mathrm{L}_{i}, \mathrm{~L}_{i}^{\prime}\right] /\left\langle\mathrm{L}_{i}^{2},\left(\mathrm{~L}_{i}^{\prime}\right)^{2}\right\rangle & \text { otherwise. }\end{cases}
$$

Note that these have bases $\left\{\mathrm{e}_{v_{i}-1}, \mathrm{~L}_{i}\right\}$ and $\left\{\mathrm{e}_{v_{i}-1}, \mathrm{~L}_{i}, \mathrm{~L}_{i}^{\prime}, \mathrm{L}_{i} \mathrm{~L}_{i}^{\prime}\right\}$, respectively. Also, the loops $\mathrm{L}_{i}$, for $\mathrm{p} \mid i$, and $\mathrm{L}_{i} \mathrm{~L}_{i}^{\prime}$, otherwise, are central since only the idempotent $\mathrm{e}_{v_{i}-1}$ does not annihilate them.

But there are more central elements. To define them, let $c(j)$ denote the set of indexes of the $j$ th column, read left to right. For example, $c(2)=\{\ldots,-8,-2,2,8, \ldots\}$ for $\mathrm{p}=5, c f$. (5-5). Then we sum row loops over their column $r(j)$, i.e.

$$
\mathrm{L}_{c(j)}=\sum_{i \in c(j)} \mathrm{L}_{i} \in \overline{\mathrm{Z}}^{G_{2} T} .
$$

We have $\mathrm{L}_{c(j)} \in Z\left(\overline{\mathrm{Z}}^{G_{2} T}\right)$ : That $\mathrm{L}_{c(j)}^{\prime}$ commutes with all idempotents is clear. Moreover, each vertical arrow annihilates $\mathrm{L}_{c(j)}^{\prime}$ from both sides and each horizontal arrow transports a loop $\mathrm{L}_{i}$ to its neighbor, illustrated as

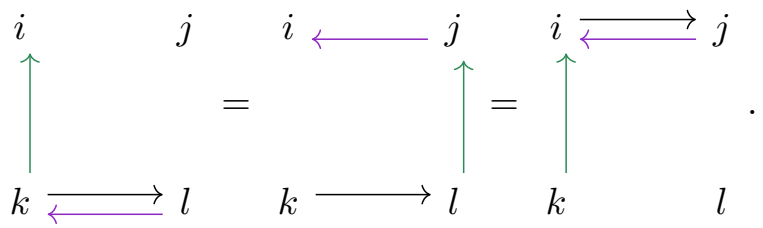


Furthermore, a bit more thought (using arguments as in Section 3) proves that the central elements which we have identified, i.e.

$$
\mathrm{L}_{i} \text {, for } \mathrm{p} \mid i, \quad \mathrm{~L}_{i} \mathrm{~L}_{i}^{\prime} \text {, otherwise, } \quad \mathrm{L}_{r(j)}^{\prime} \text {, for } j \in\{1, \ldots, \mathrm{p}-1\} \text {, }
$$

form a basis of $Z\left(\bar{Z}^{G_{2} T}\right)$, and it remains to calculate the relations among these. We already know the relations among the elements $\mathrm{L}_{i}$ and $\mathrm{L}_{i} \mathrm{~L}_{i}^{\prime}$, see (5-6). Further, we have

$$
\mathrm{L}_{c(j)} \mathrm{L}_{c(k)}=0, \quad \mathrm{~L}_{c(j)} \mathrm{L}_{i}=0=\mathrm{L}_{i} \mathrm{~L}_{c(j)}, \text { for } \mathrm{p} \mid i, \quad \mathrm{~L}_{c(j)} \mathrm{L}_{i} \mathrm{~L}_{i}^{\prime}=0=\mathrm{L}_{i} \mathrm{~L}_{i}^{\prime} \mathrm{L}_{c(j)} .
$$

This concludes the proof.

\section{REFERENCES}

[An19] H.H. Andersen. Tilting modules and cellular categories. J. Pure Appl. Algebra, 224(9):106366, 2020. URL: https://arxiv.org/abs/1912.00817, 10.1016/j.jpaa.2020.106366.

[AST18] H.H. Andersen, C. Stroppel, and D. Tubbenhauer. Cellular structures using $\mathrm{U}_{q}$-tilting modules. Pacific J. Math., 292(1):21-59, 2018. URL: https://arxiv.org/abs/1503.00224, doi:10.2140/pjm. 2018. 292.21.

[AT17] H.H. Andersen and D. Tubbenhauer. Diagram categories for $\mathbf{U}_{q}$-tilting modules at roots of unity. Transform. Groups, 22(1):29-89, 2017. URL: https://arxiv.org/abs/1808.08022, doi:10.1007/ s00031-016-9363-z.

[Don93] S. Donkin. On tilting modules for algebraic groups. Math. Z., 212(1):39-60, 1993. doi:10.1007/ BF02571640.

[TW19] D. Tubbenhauer and P. Wedrich. Quivers for SL(2) tilting modules. Preprint. URL: https://arxiv . org/abs/1907.11560.

[Wil17] G. Williamson. Algebraic representations and constructible sheaves. Jpn. J. Math., 12(2):211-259, 2017. URL: https://arxiv.org/abs/1610.06261, doi:10.1007/s11537-017-1646-1.

D.T.: Institut für Mathematik, Universität Zürich, Winterthurerstrasse 190, Campus Irchel, Office Y27J32, CH-8057 Zürich, Switzerland, www.dtubbenhauer.com

Email address: daniel.tubbenhauer@math.uzh.ch

P.W.: Mathematical Sciences Research Institute, 17 Gauss Way, Berkeley, CA 94720, USA, PAUL.WEDRICH.AT

Email address: p.wedrich@gmail.com 